\title{
SPATIALLY LOCALIZED STRUCTURES IN DIBLOCK COPOLYMER MIXTURES*
}

\author{
KARL B. GLASNER ${ }^{\dagger}$
}

\begin{abstract}
Above the critical temperature for the order-disorder transition, diblock copolymer melts have been observed to exhibit localized structures that exist within the homogeneous mixture. This paper uses an Ohta-Kawasaki-type density functional to explore this regime. Spatially localized peak-shaped equilibria are studied in one, two, and three dimensions, corresponding to amphiphilic bilayers, cylindrical micelles, and spherical micelles, respectively. A combination of rigorous estimates, asymptotic analysis, and numerical computation is used to characterize solutions and the regime where they exist. The interaction of superpositions of these solutions is studied by a perturbation analysis and shows how steady multipeak configurations can be achieved. Evidence is found for a secondary bifurcation slightly below the spinodal instability threshold, beyond which self-replication phenomena are observed. Dynamics in two dimensions are also illustrated, suggesting other mechanisms for instability and growth.
\end{abstract}

Key words. diblock copolymer, micelle, density functional theory, self-replication

AMS subject classifications. 82D60, 35K55

DOI. $10.1137 / 080743913$

1. Introduction. Diblock copolymers are linear chain molecules composed of two distinct subchains. At low temperatures, repulsion between subchains can result in phase segregation, but macroscopic segregation cannot occur because of covalent bonding between subchains. This incomplete segregation can give rise to a variety of stable microstructures (see, e.g., $[1,11,14,24]$ ), which are typically characterized as spatially periodic patterns whose domain boundaries closely resemble minimal surfaces, such as spheres, cylinders, and gyroids [39].

Outside of the regime where mixtures are unstable to spinodal decomposition, the situation for copolymers is more complicated than simple binary systems, such as those described by the Cahn-Hilliard theory [5]. In addition to a stable homogeneous state, there are other observed behaviors. Physical experiments have identified inhomogeneous states above the critical temperature, such as sparse arrangements of micellar structures $[35,36,43]$ and anisotropic lamellar grains [15]. This paper seeks to investigate this regime and its dynamics using density functional theory and the corresponding gradient flow dynamics.

Theoretical work on the disordered regime has focused on spherical micelles. Dormidontova and Lodge [9] use Semenov's strong segregation theory [37] to predict the concentration of micelle assemblies. Their theory predicts that the number of micelles increases rapidly below a "critical micelle temperature" (CMT), which is greater than the temperature at which a homogeneous mixture is spinodally unstable. Wang, Wang, and Yang [42] use self-consistent field theory calculations to find the density profile of individual spherical micelles and evaluate their excess free energy. They find that micelles exist (within the mean field approximation) for volume frac-

${ }^{*}$ Received by the editors December 15, 2008; accepted for publication (in revised form) January 19, 2010; published electronically March 24, 2010. This work was supported under NSF award DMS0405596.

http://www.siam.org/journals/siap/70-6/74391.html

${ }^{\dagger}$ Department of Mathematics, University of Arizona, Tucson, AZ 85721 (kglasner@math.arizona. edu). 
tions $\lesssim .22$ and only below another critical temperature that they call the "micelle disassociation temperature," which is higher than the CMT.

This paper uses density functional theory to study energy-minimizing equilibria and dynamics in a region of parameter space where the homogeneous state is stable. We demonstrate the existence of not only spherically symmetric minima, but also oneand two-dimensional analogues which correspond to amphiphilic bilayers and "cylindrical" micelles, respectively. These exist only above a bifurcation point (relative to the interaction strength parameter) and below a secondary bifurcation that occurs before the spinodal instability threshold. We show how these isolated structures can interact dynamically by using a perturbation scheme to obtain a finite dimensional reduction. We also exhibit numerical computations where structures are created by front propagation or grow into long filaments.

Our work, which is strictly about binary $(A-B)$ copolymer mixtures, should be contrasted with the case of copolymer-homopolymer or copolymer-solvent mixtures. There, isolated micelles and bilayers are generically expected to form in the phaseseparated regime $[16,40,41]$, since the solvent or homopolymer can fill the adjacent volume. For pure diblock copolymers, on the other hand, the region between micelletype structures must be filled with a homogeneous mixture, which may or may not be unstable.

This paper's results have some qualitative similarity to a variety of systems that arise in the reaction-diffusion literature $[13,21,27,45]$. Pattern formation in variational models similar to ours has been studied by Muratov [26]. In that paper, steady state solutions and their dynamics were explored, principally focusing on the sharp interface limit and short-ranged nonlocal interaction energy. In contrast, the current study deals solely with the long-ranged interaction particular to block copolymers and exploits the finite size of the diffuse interface.

The paper is organized as follows. Section 2 reviews established modeling paradigms for block copolymer mixtures and elaborates on the density functional model used here. Section 3 discusses single-peaked equilibria in one and several dimensions. These are used as building blocks for multipeaked solutions that are discussed in section 4. A more general discussion of dynamical issues is given in section 5 .

2. Models for $\boldsymbol{A}-\boldsymbol{B}$ block copolymers. Theoretical models of block copolymer melts fall roughly into three categories. The earliest of these were phenomenological descriptions based on Landau theory, such as that due to Brazovskii [4]. While capturing some of the essential aspects of pattern formation, they do not make quantitative predictions about the phase diagram.

Another approach which has become widely utilized is the self-consistent (or mean-) field approximation of the Gaussian chain ensemble with Flory-type interaction $[17,19,23,25]$. In this theory, there are two nondimensional parameters: the volume fraction of $A$ monomers $f$, and the interaction strength $\chi N$, which inversely corresponds to temperature. Here $\chi$ represents the (Flory) interaction energy (measured in units of $k T$ ), and $N$ is the degree of polymerization.

Expressions for the free energy in terms of monomer volume fraction (density functional theory) make up a third class of models. The most commonly invoked formulation was derived by Ohta and Kawasaki [30], having the form

$$
F(\phi)=\int \frac{a}{2}|\nabla \phi|^{2}+g(\phi ; \chi N) d x+\frac{\gamma}{2} \iint G\left(x-x^{\prime}\right)[\phi(x)-f]\left[\phi\left(x^{\prime}\right)-f\right] d x d x^{\prime}
$$

Here $\phi$ is the volume fraction of $A$ monomers (whereby the standard incompressibil- 
ity assumption $1-\phi$ is the $B$-monomer fraction), $G$ is the usual free-space Green's function for the Laplacian, and $g$ is a double-well energy. Although there is no first principles derivation for this functional, the details in this expression can be loosely tied to self-consistent field theory by a series of approximations [7]. Various refinements and extensions of this type of model have been proposed [3,31,40] but largely keep the structure of the functional intact. In particular, it has been proposed to have coefficients depend on some local average of volume fraction rather than the global fraction $f$ [31], but we do not take that course here. In the work of Uneyama and Doi [40], the free energy is roughly of this form, except that $\phi$ must be interpreted as an order parameter equal to the square root of the density.

The main advantage of density functional theory is that it is more amenable to analytic and numerical calculation than self-consistent field theories. Dynamical versions are also easy to construct as gradient descent of the free energy [28]. We take the viewpoint that while these models may not be entirely quantitatively accurate, they lend substantial insight into the self-organization of copolymer mixtures and retain the salient features of the phase diagram.

2.1. Calibration of the density functional. Much of this paper is concerned with general mathematical properties of (2.1), and most results do not hinge on the specific choices of potential $g$ or constants $a, \gamma$ in (2.1). On the other hand, it is scientifically useful to have a model whose consequences can be quantitatively interpreted and compared. This is done here by calibrating the constitutive quantities in the density functional to a set of physically motivated criteria.

We will limit the form of the double-well potential to

$$
g(\phi)=h(\phi)+\chi N \phi(1-\phi)
$$

where $h$ is yet unknown (this choice implies a gauge for the free energy in units of $k T$ ). Lengths will be measured relative to the radius of gyration $R_{g}=b \sqrt{N / 6}$, where $b$ is the Kuhn length [11].

The functional (2.1) therefore has three quantities to be specified: the coefficients $a, \gamma$ and the potential $h$. Rather than constructing an expression from first principles, an alternative approach is adopted here, which is to choose the constitutive quantities to make certain key physical features coincide with a chosen benchmark. The criteria which are adopted are as follows:

(a) The diffuse interface width is adjusted to fit available theory and experiment.

(b) Since this study is concerned with behavior near the spinodal point, the model should reproduce a prescribed spinodal (stability) curve $\chi N=s(f)$.

(c) The marginally stable wavenumber $q^{*}(f)$ is also reproduced according to a prescribed benchmark.

For criterion (a), it has been noted elsewhere [31,38] that the interfacial energy for strong segregation $\chi N \gg 1$ should not depend on the composition $f$ and should scale as $(\chi N)^{-1 / 2}$. In the large $\chi N$ limit, a one-dimensional interface is described by the Euler-Lagrange equation

$$
\phi_{x x}+\frac{2 \chi N}{a} \phi=\lambda
$$

where $\lambda$ is a Lagrange multiplier. The unique solution connecting the states $\phi=0,1$ is

$$
\phi=\frac{1}{2}(\cos (\pi x / \xi)+1), \quad \xi=\pi \sqrt{\frac{a}{2 \chi N}}
$$


where $\xi$ is the interface width.

Helfand and Tagami [18] compute the interface width to be $2 b / \sqrt{6 \chi}$, which in units of the $R_{g}$ gives $\xi=2 / \sqrt{\chi N}$. Semenov [38] carefully compares this prediction with experiments and develops corrections to their theory, which underestimates the interface width by a factor of about 1.7. When required, we will use the value $\xi=$ $3.4 / \sqrt{\chi N}$ so that $a \approx 2.3$.

In principle, criteria (b) and (c) can be met for any reasonable specification of spinodal curve and marginally unstable wavenumber. For purposes of specificity, we shall choose the constitutive quantities $h$ and $\gamma$ to be consistent with the widely cited theory of Leibler [23], which is based on a Gaussian chain ensemble. In this theory, the spinodal instability is identified with a singularity in the (Fourier transformed) correlation function obtained from the random phase approximation. From [23], this function was computed as

$$
\begin{aligned}
S(q) & =\frac{N}{\Phi\left(q^{2} ; f\right)-2 \chi N}, \\
\Phi(x ; f) & =\frac{g_{1}(1, x)}{g_{1}(1-f, x) g_{1}(f, x)-\frac{1}{4}\left[g_{1}(1, x)-g_{1}(f, x)-g_{1}(1-f, x)\right]^{2}},
\end{aligned}
$$

where $g_{1}(f, x)=2(f x+\exp (-f x)-1) / x^{2}$ and the wavenumber $q$ is measured in units of $1 / R_{g}$. The instability therefore occurs where two conditions are met, namely,

$$
\chi N=\Phi\left(q^{*}(f)^{2} ; f\right) / 2, \quad \Phi_{x}\left(q^{*}(f)^{2} ; f\right)=0 .
$$

The latter equation implicitly determines $q^{*}(f)$, and the former gives the spinodal curve $s(f)=\Phi\left(q^{*}(f)^{2} ; f\right) / 2$. The second variation of $(2.1)$ about $\phi=f$ gives a bilinear form in test functions $\psi$ :

$$
\int \frac{a}{2}|\nabla \psi|^{2}+\frac{1}{2} g_{\phi \phi}(f ; \chi N) \psi^{2} d x+\frac{\gamma(f)}{2} \iint G\left(x-x^{\prime}\right) \psi(x) \psi\left(x^{\prime}\right) d x d x^{\prime} .
$$

Positive definiteness that indicates stability is implied if for all wavenumbers $q$

$$
a q^{4}+g_{\phi \phi}(f ; \chi N) q^{2}+\gamma(f) \equiv \sigma(q) \geq 0 .
$$

The most unstable wavenumber satisfies $d \sigma / d \phi=0$, which gives

$$
q^{*}(f)=\sqrt{\frac{-g_{\phi \phi}(f ; \chi N)}{2 a}} .
$$

The instability is located by $\sigma\left(q^{*}\right)=0$; using (2.2) this leads to the critical value for $\chi N$ :

$$
s(f)=\sqrt{a \gamma(f)}+h^{\prime \prime}(f) / 2 .
$$

The corresponding most unstable wavelength is given by

$$
q^{*}(f)=\left(\frac{\gamma(f)}{a}\right)^{\frac{1}{4}} .
$$

Thus, to coincide with Leibler's result, $\gamma=a q^{*}(f)^{4}$. Using (2.10), it follows that $h$ solves

$$
\frac{\partial^{2} h}{\partial \phi^{2}}=2(s(\phi)-\sqrt{a \gamma(\phi)})
$$




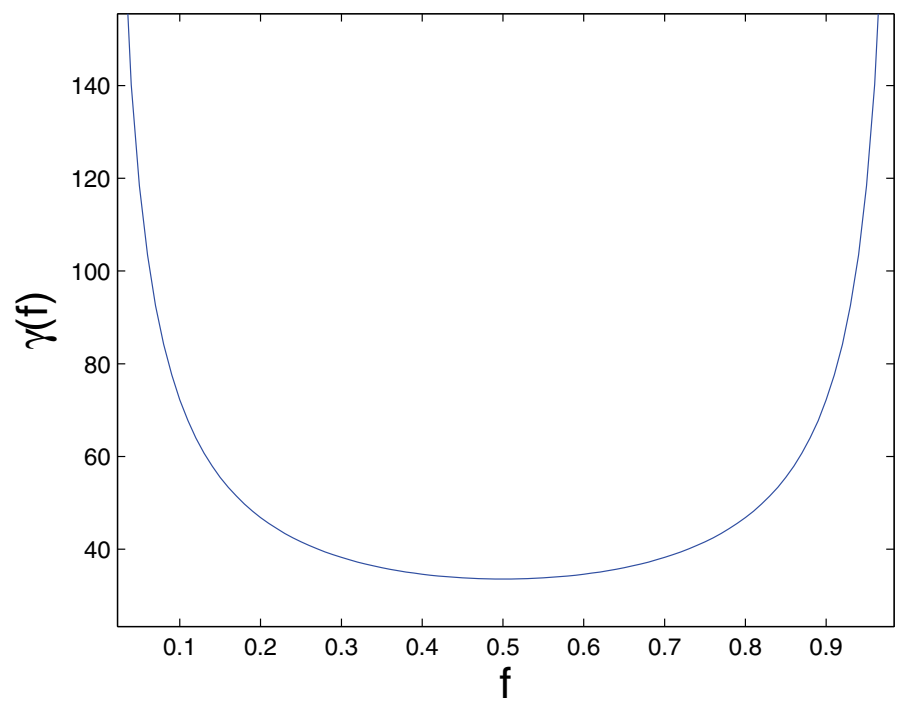

FIG. 1. The coefficient $\gamma$ as a function of composition $f$.

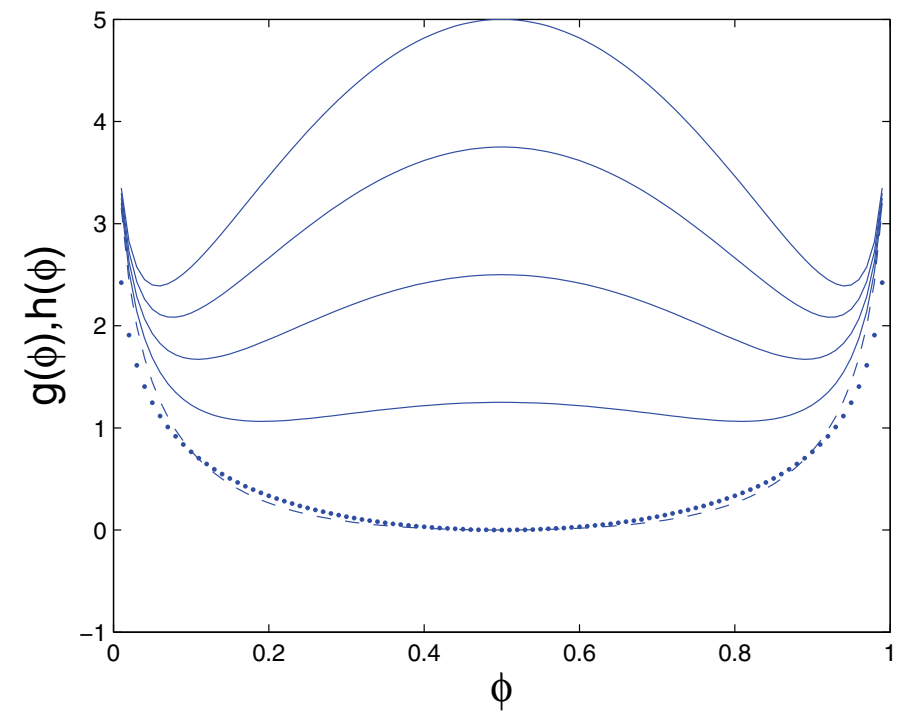

FIG. 2. The double well potential (solid) $g(\phi ; \chi N)$ for values of $\chi N=5,10,15,20$, and the potential $h(\phi)$ (dashed) together with its analytic approximation (dotted).

This equation has a unique solution symmetric about $\phi=1 / 2$ that has zero minimum. Note that $\gamma$ depends on the volume fraction here, although it will occasionally prove insightful to treat it as an independent parameter.

To make further progress, $h$ must be computed numerically. We have done this by first solving (2.6) to determine $s(f)$ and $q^{*}(f)$, and then using a straightforward finite difference method to determine $h$ from (2.12). It is useful to look for an analytical 
approximation by least squares; the form

$$
h \approx A(\ln (\phi)+\ln (1-\phi))+B \phi(1-\phi)+C \phi^{2}(1-\phi)^{2}+D
$$

with the values $A=-1.3, B=0.23, C=6.05$, and $D=1$ gives a very good fit (Figure 2 ) over the relevant range of $\phi$. This analytical expression is used for numerical simulations in what follows. We find that it predicts the correct stability threshold within a few percent. Figure 1 shows the parameter $\gamma$ as a function of $f$. Figure 2 plots the potential $g$ at various values of $\chi N$, as well as the function $h$ and its analytical approximation.

2.2. Dynamics. A natural way of imparting dynamics to (2.1) is via a CahnHilliard-type gradient flow. This arises from assuming a linear relationship between the chemical potential $\delta F / \delta \phi$ and the flux of material (related by a kinetic coefficient) and leads to the fourth order parabolic equation [28]

$$
\phi_{t}=-a \Delta^{2} \phi+\Delta g_{\phi}(\phi ; \chi N)+\gamma(f-\phi) .
$$

The timescale for $t$ has been chosen to absorb the kinetic coefficient. Our purpose in using (2.14) is twofold. Since stable equilibria of (2.14) are energy minimizers subject to the correct volume fraction (assuming the initial condition also satisfies this constraint), the dynamical equation represents a steepest descent method to find minimizers. We also study the dynamics of (2.14) in their own right, since they have at least a qualitative bearing on how complex equilibria form. We do not address the question of timescales, which are ultimately a function of molecular kinetics, nor do we include fluctuations, which can be relevant in the study of block copolymer evolution [12].

3. Single-peak solutions. This section deals with the fundamental question of energy-minimizing equilibria which represent a single structure embedded in an otherwise mixed, flat state. In contrast to well-known periodic equilibria that occur for unstable mixtures, the focus here is on steady solutions that decay at an exponential rate in the far field. In the language of reaction-diffusion equations, these are sometimes called pulse or "spike" [20] solutions.

3.1. One-dimensional bilayer solutions. We first investigate the existence of one-dimensional local minimizers of the functional (2.1) in the regime below the spinodal curve where the homogeneous state is stable. Projected into a three-dimensional setting, these correspond physically to flat sheets of amphiphilic bilayers. As will be demonstrated later, these structures can arise dynamically from cylindrical micelles via nonlinear perturbations.

The specific problem that is studied entails looking for exponentially decaying solutions to the steady state equation

$$
\left(-a \phi_{x x}+g_{\phi}(\phi ; \chi N)\right)_{x x}+\gamma(f-\phi)=0
$$

on an infinite domain. Precisely, we require

$$
\lim _{x \rightarrow \pm \infty} \phi=f .
$$

For numerical purposes, it suffices to use Neumann boundary conditions with a domain much larger than the size of the spatially localized perturbation of the flat state. 
Note that by integration of (3.1), one immediately satisfies the constraint on the compositional volume

$$
\int_{-\infty}^{\infty}(\phi-f) d x=0
$$

One can ascertain the structure of solutions for large $|x|$ by linearizing (3.1) about $\phi=f$. Supposing $(\phi-f) \sim \exp (Q x)$, where $Q$ may be complex, one gets the relationship

$$
a Q^{4}-g_{\phi \phi}(f ; \chi N) Q^{2}+\gamma=0
$$

Thus

$$
Q^{2}=\frac{g_{\phi \phi}(f ; \chi N) \pm \sqrt{g_{\phi \phi}(f ; \chi N)^{2}-4 a \gamma}}{2 a} .
$$

There are three possibilities for the far field behavior. If $g_{\phi \phi}(f ; \chi N)$ is sufficiently positive, then $g_{\phi \phi}^{2}-4 a \gamma>0$, and the roots $Q$ are real, implying strict exponential growth or decay. Only the latter is admissible according to (3.2), so the far field behavior is characterized by

$$
\phi \approx f+c_{1} \exp \left(\mp Q_{1} x\right)+c_{2} \exp \left(\mp Q_{2} x\right), \quad x \rightarrow \pm \infty .
$$

If $\left(g_{\phi \phi}(f ; \chi N)\right)^{2}<4 a \gamma$, the roots $Q$ are strictly complex. From here on, $Q$ will denote the root with positive real and imaginary parts. It follows that

$$
\phi \approx f+\exp (\mp[\operatorname{Re} Q] x)\left(c_{1} \cos ([\operatorname{Im} Q] x)+c_{2} \sin ([\operatorname{Im} Q] x)\right), \quad x \rightarrow \pm \infty .
$$

One can observe that as $\chi N$ increases toward the spinodal stability threshold $s(f)$, the discriminant in (3.5) becomes small, and thus $Q$ necessarily has small real part (note that $g_{\phi \phi}<0$ there). Therefore, solutions near the instability threshold $\chi N=s(f)$ will have highly oscillatory tails.

Beyond the instability threshold, inequality $(2.8)$ does not hold for the most unstable wavelength (2.11), which implies that $\left(g_{\phi \phi}(f ; \chi N)\right)^{2}>4 a \gamma$ and $g_{\phi \phi}(f ; \chi N)<0$. This means that exponential decaying solutions are impossible in this regime; small amplitude stationary solutions (if these exist at all) must necessarily oscillate at infinity.

3.1.1. Construction via asymptotics. To lend insight into the nature of equilibria, we allow $\gamma$ to be independent of $f$ and utilize a matched asymptotic construction for small $\gamma$. This is meaningful from a mathematical perspective, since in the absence of bifurcations (which are discussed below), these solutions can be continued to large $\gamma$. The advantage of this approximation is that it reveals the architecture of the solution as consisting of two interfaces enclosing a core which is rich in $A$ monomers. The construction crucially depends on the core being larger than the interface width, which in general can be achieved by making $f$ smaller or $\chi N$ larger (but below the threshold for instability). This existence criteria is corroborated and quantified in later subsections for arbitrary $\gamma$; in particular, there is a minimum value of $\chi N$ for existence of steady solutions at which a saddle-node bifurcation occurs. Wang, Wang, and Yang [42] find essentially the same behavior in their self-consistent field theory calculations. Their "micelle disassociation temperature" corresponds to this bifurcation. 
When $\gamma$ is set to zero, the instability threshold is $s(f)=h^{\prime \prime}(f) / 2$ and coincides with the spinodal curve of the usual Cahn-Hilliard equation. Therefore, we must restrict $\chi N$ to that regime, which is equivalent to assuming that

$$
g_{\phi \phi}(f ; \chi N)>0 .
$$

We suppose that, in addition, $g(\phi)$ has two minima $\phi_{ \pm}$where $\phi_{-}<f<\phi_{+}$. This guarantees the ability to construct the diffuse interface that encloses the core region.

Symmetry is assumed by imposing reflective boundary conditions at $x=0$. There are three regions with different scalings: the core, the interface, and the corona (i.e., exponentially decaying tail). In the corona and core regions, lengths scale as $\gamma^{-1 / 2}$, whereas the interface width is independent of $\gamma$.

The interface is described at leading order in $\gamma$ as a solution to

$$
\left(-a \phi_{x x}+g_{\phi}(\phi ; \chi N)\right)_{x x}=0 .
$$

Matching to outer regions with a larger length scale implies that solutions approach constants at large $|x|$, i.e., $\lim _{x \rightarrow \pm \infty} \phi_{x}=0$. The solution describing the interface is the well-known internal layer for the classical Cahn-Hilliard equation [33], which in particular satisfies

$$
\lim _{y \rightarrow \pm \infty} \phi=\phi_{ \pm}, \quad \phi_{-}<f<\phi_{+}, \quad \phi_{ \pm} \text {are minima of } g
$$

(these are consistent with the standard Maxwell construction). For $\chi N \gg 1$ and the calibrated potential, these values are roughly $\phi_{-}=0$ and $\phi_{+}=1$.

The stretched coordinate $z=x / \gamma^{-1 / 2}$ is used in the core and corona regions, which correspond to the intervals $\left[0, z^{*}\right)$ and $\left(z^{*}, \infty\right)$, respectively. The scaled core width $z^{*}$ will be determined by using the global constraint (3.3), which leads to the requirement

$$
\int_{z^{*}}^{\infty}(f-\phi(z)) d z=\int_{0}^{z^{*}}(\phi(z)-f) d z .
$$

In the rescaled regions, dominant balance of terms in the steady state equation leads to

$$
\left[g_{\phi}(\phi, \chi N)\right]_{z z}-(\phi-f)=0
$$

subject to boundary and matching conditions

$$
\phi_{z}(0)=0, \quad \lim _{z \rightarrow z^{*}-} \phi=\phi_{+}, \quad \lim _{z \rightarrow z^{*}+} \phi=\phi_{-}, \quad \lim _{z \rightarrow \infty} \phi=f .
$$

By the requirement (3.8), it follows that $g_{\phi \phi}>0$ on the interval $[0, f]$. It is also true on some interval $\left[\phi_{i}, 1\right]$ containing $\phi_{+}$, where $\phi_{i}$ is an inflection point of $g()$. This allows (3.12) to be converted to a standard integrable Hamiltonian by the Legendre transform $g^{*}(\psi)=\max _{\phi}[\phi \psi-g(\phi ; \chi N)]$. Properly speaking, there are two such transforms, one for the convex interval around $\phi_{-}$and one for the convex interval around $\phi_{+}$, but the distinction will be clear from the context. In the new variable defined by $\psi=d g(\phi) / d \phi$, equation (3.12) becomes

$$
\psi_{z z}+\left(f-\frac{d g^{*}(\psi)}{d \psi}\right)=0
$$



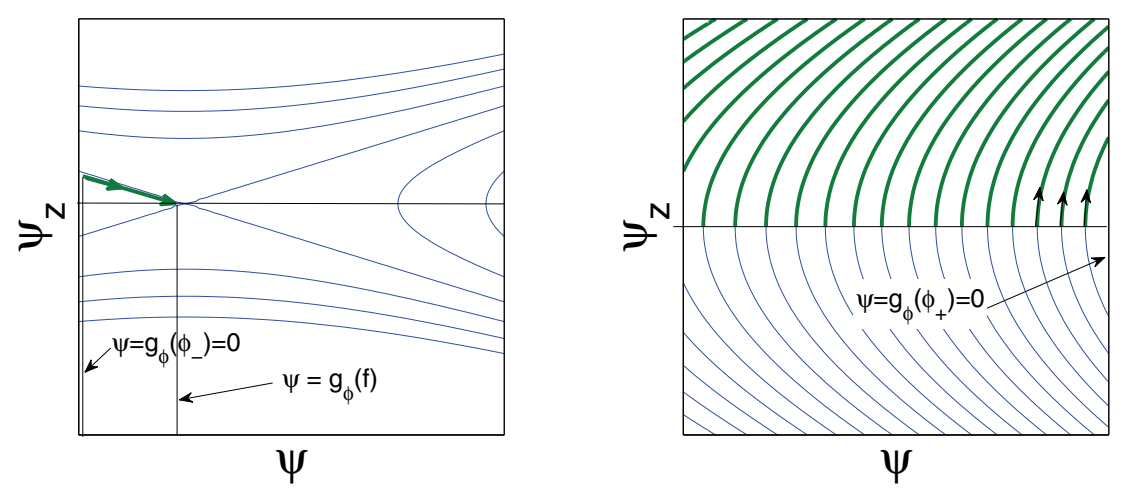

FIG. 3. Phase portrait sketches for system (3.12), showing contours of conserved quantity $H\left(\psi, \psi_{x}\right)=\psi_{x}^{2}+f \psi-g^{*}(\psi)$. Left: Curve extending from $\psi=g_{\phi}\left(\phi_{-}\right)$to the saddle along the stable manifold corresponds to the solution on $\left(z^{*}, \infty\right)$. Right: There are many trajectories which extend from the $\psi_{x}=0$ axis up to the line $\psi=g_{\phi}\left(\phi_{+}\right)=0$. The relevant trajectory is selected by (3.11).

This equation is satisfied on each interval $\left[0, z^{*}\right)$ and $\left(z^{*}, \infty\right)$, subject to the transformed boundary conditions

$\psi_{z}(0)=0, \quad \lim _{z \rightarrow z^{*}-} \psi=g_{\phi}\left(\phi_{+}\right)=0, \quad \lim _{z \rightarrow z^{*}+} \psi=g_{\phi}\left(\phi_{-}\right)=0, \quad \lim _{z \rightarrow \infty} \psi=g_{\phi}(f) \equiv \psi_{f}$.

The Hamiltonian for this system is $H\left(\psi, \psi_{x}\right)=\psi_{x}^{2}+f \psi-g^{*}(\psi)$. By virtue of $\partial H / \partial \psi\left(\psi_{f}, 0\right)=0$ and $\partial H^{2} / \partial \psi^{2}\left(\psi_{f}, 0\right)<0$, the point $\left(\psi_{f}, 0\right)$ is a saddle. The solution on $\left(z^{*}, \infty\right)$ therefore corresponds to part of the stable manifold in the upper half-plane (see Figure 3). By inversion, $\phi$ may be determined in principle, and this completely specifies the left-hand side of (3.11).

For the problem on $\left[0, z^{*}\right]$, there is a family of solutions to (3.12) satisfying the boundary conditions (3.15). These correspond to the various trajectories that emanate from the $\psi$-axis in the Hamiltonian phase plane (Figure 3 ) and are parameterized by the initial condition $\psi(0) \in\left[g_{\phi}\left(\phi_{i}\right), 0\right]$. Note that as $\psi(0) \rightarrow 0$ (i.e., $\left.\phi(0) \rightarrow \phi_{+}\right)$, the trajectories become short, and the right-hand side of (3.11) goes to zero. Therefore, we may expect to have solutions provided the left-hand side of (3.11) is not too big. The estimate (which is derived in the appendix)

$$
\int_{z^{*}}^{\infty}(f-\phi(z)) d z \leq C\left(f-\phi_{-}\right), \quad C \text { independent of } f,
$$

shows that as long as $f$ is close to $\phi_{-}$, it will be possible to satisfy (3.11). Computations and other analytical evidence developed later reveal that existence should not be expected for more general values of $f$, however.

From a quantitative perspective, the exact solutions to (3.12) and (3.11) are simply too complicated to lend much insight. As an example, consider the quadratic approximations for the potential

$$
g(\phi) \approx \frac{1}{2} \beta^{2}\left(\phi-\phi_{ \pm}\right)^{2}, \quad \beta^{2} \equiv g^{\prime \prime}\left(\phi_{ \pm}\right)
$$


near each well $\phi=\phi_{ \pm}$. The solutions in each region can be computed explicitly as

$$
\phi= \begin{cases}f+\left(\phi_{-}-f\right) \exp \left(-\left(z-z^{*}\right) / \beta\right), & z>z^{*} \\ f+\left(\phi_{+}-f\right) \cosh (z / \beta) / \cosh \left(z^{*} / \beta\right), & 0<z<z^{*}\end{cases}
$$

The volume constraint (3.11) becomes

$$
\tanh \left(z^{*} / \beta\right)=\frac{f-\phi_{-}}{\phi_{+}-f}
$$

which can be solved to determine the core width $z^{*}$, provided $f \leq\left(\phi_{-}+\phi_{+}\right) / 2=1 / 2$. For small $A$-monomer fractions one can approximate further $\phi_{+}-f \approx 1$ and linearize to get the core width approximation

$$
z^{*} \approx \beta\left(f-\phi_{-}\right)
$$

For $f=0.1$ and $\chi N=50$, using the calibrated potential, one has $\phi_{-} \approx 0.026, \beta \approx 43$ so that the unscaled core width is $\approx 3.2 \gamma^{-1 / 2}$. This is in reasonable agreement with numerical solutions (see Figure 5), even when $\gamma$ is large.

3.1.2. Rigorous results on existence and nonexistence. Unlike the spinodally unstable regime, where patterns and dynamic behavior have been studied at length, the question of nontrivial behavior below threshold is more delicate. Before proceeding to develop further numerical and analytical evidence of equilibrium and nonequilibrium behavior, we state some rigorous estimates to establish where relaxation to the flat state is and is not expected. The motivation for this is both to ensure that nontrivial behavior can occur in the spinodally stable regime and to provide guidance as to where this occurs. The results are not specific to the calibrated potential in (2.1) and should apply to qualitatively similar models. The proofs of the following propositions are deferred to the appendix.

First we estimate a region where only the constant (homogeneous) state is a minimizer.

Proposition 3.1. If

$$
\chi N \leq \sqrt{\gamma a}+\frac{1}{2} \inf _{\phi \in[0,1]} h^{\prime \prime}(\phi),
$$

then the only minimizer to (2.1) satisfying the volume fraction constraint (3.3) is the constant (homogeneous) state $\phi \equiv f$.

This result is a slight strengthening of the result of Choksi, Peletier, and Williams [6], who show that $\phi \equiv f$ is the global minimum under the same hypothesis. The question for us is a little different, as the later numerical results indicate that local minimizers can exist that have energy greater (as well as less) than the homogeneous state $\phi \equiv f$.

The second result provides information on the regime where solutions may not relax to the homogeneous state $\phi=f$. The idea is to construct an approximate "bilayer" solution, optimized over the core and interface widths. The main technicality is to show that the relative bulk energy benefit (the $g()$-term) in the corona region is greater than the penalty paid by the surface and nonlocal energies. This does not guarantee the existence of steady peak-type solutions but shows that a certain class of initial conditions will do something other than relax to a constant. 


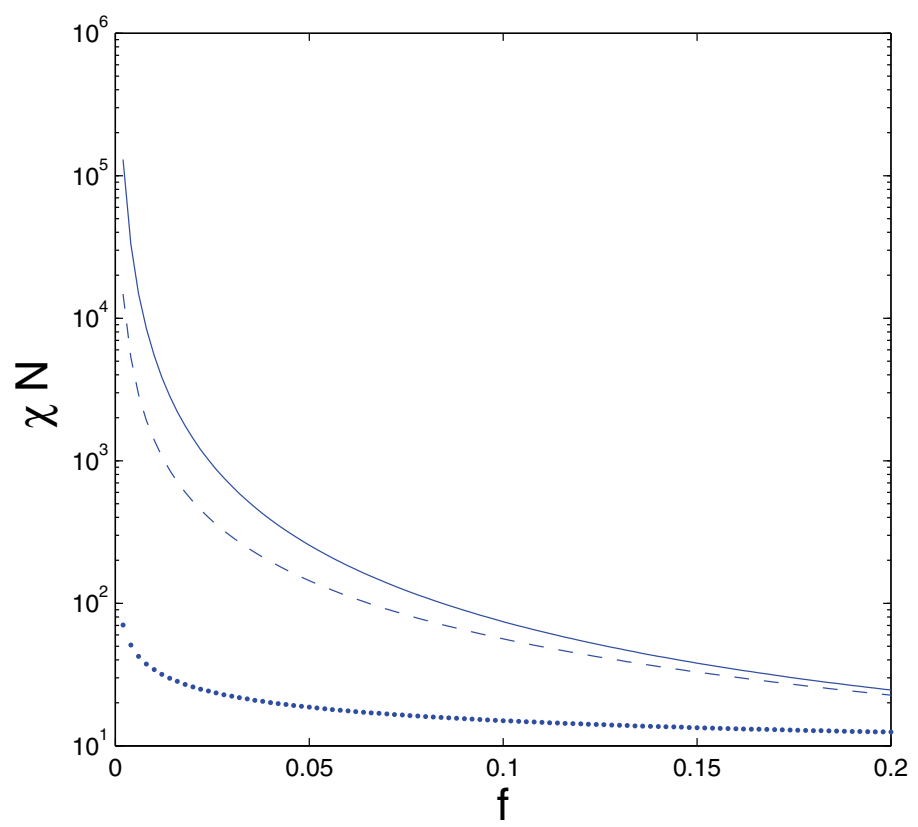

FIG. 4. Rigorous bounds relative to the phase diagram. The dotted (lower) curve is the bound (3.21), below which nonconstant solutions cannot exist. The middle dashed curve is the minimum value of $\chi N$ that satisfies (3.22) with $C=1$, above which nonconstant solutions may persist. The solid line is the spinodal curve above which the flat state is unstable.

Proposition 3.2. Suppose that $\chi N, a, \gamma$, and $f$ are chosen so that

$$
\frac{4}{3} \sqrt{\frac{\sigma_{2}^{3}\left(f-\phi_{-}\right)}{C \gamma}} \geq \frac{\sqrt{3}}{3} \sqrt{a \sigma_{1}}+\frac{\sqrt{3}}{8} \sqrt{\frac{a^{2} \gamma\left(f-\phi_{-}\right)^{5}}{\sigma_{2}}} ;
$$

then there exist states satisfying the volume fraction constraint (3.3) with energy less than $\phi \equiv f$. Here $\phi_{ \pm}$are minima of $g(\phi), \sigma_{1}$ is chosen so that $g(\phi) \leq \sigma_{1} \phi(1-\phi)$ when $\phi \in\left[\phi_{-}, \phi_{+}\right], \sigma_{2}$ is chosen so that $g(\phi)-g(f) \leq \sigma_{2}(\phi-f)$ when $\phi<f$, and the constant $C \rightarrow 1$ as $f \rightarrow 0$.

Although (3.22) is complicated, is can readily be evaluated numerically as follows. For each $f$, the minimum value of $\chi N$ is determined which satisfies (3.22). The constants $\sigma_{1,2}$ can be determined for a particular $\chi N$ as

$$
\begin{aligned}
\sigma_{1} & =\max _{\left[\phi_{-}, \phi_{+}\right]} \frac{g(\phi)}{\phi(1-\phi)}, \\
\sigma_{2} & =\max _{\left[\phi_{-}, f\right]} \frac{g(\phi)-g(f)}{\phi-f} .
\end{aligned}
$$

Using the calibrated potential and constants as described in section 2.1, the estimates (3.21) and (3.22) are plotted in Figure 4. The main observation is that nontrivial minimizers will exist below the spinodal curve over a range of $\chi N$ for sufficiently small volume fractions. 


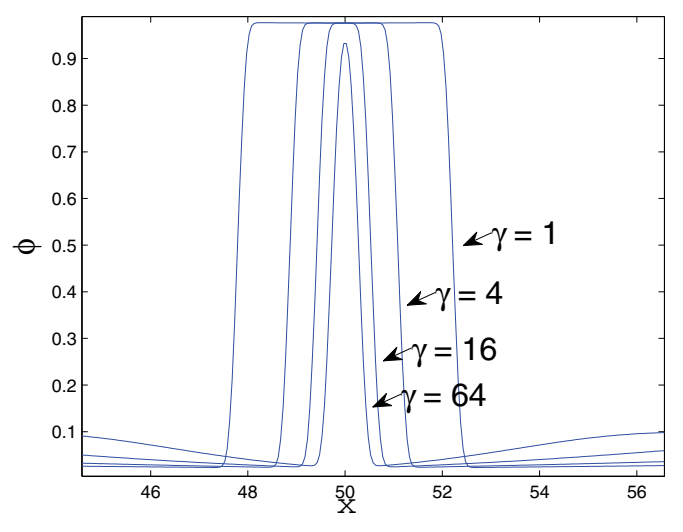

FIG. 5. Solutions for various values of $\gamma$ for $f=.1, \chi N=50$. The computational domain was $[0,100]$, but only the core structure is shown.

3.1.3. Numerical results. To make further progress in understanding the nature of steady one-dimensional solutions, the dynamical equations were numerically evolved until a steady state was reached. The initial data was $\phi=f$ for $|x|>1$ and $\phi=0.9$ for $|x|<1$. This large perturbation was usually sufficient to drive the system to a nonconstant equilibrium.

To illustrate the asymptotics of small $\gamma$ and how they connect to solutions at large $\gamma$, we chose a particular scenario where $f=0.1, \chi N=50$. Figure 5 shows steady solutions for various values of $\gamma$. The scaling of the core and corona widths as $\chi N^{-1 / 2}$ is evident. As $\gamma$ is increased further, the core width becomes smaller than the interface width (which is independent of $\gamma$ ), and bilayer-type solutions cease to exist.

Another set of computations shows how the solutions' existence depends on $\chi N$. To characterize solutions, we measured the core width, defined as the length of the interval where $\phi>1 / 2$. The computation was carried out for $f=.1$ and $\gamma=71$, which corresponds to the calibrated value discussed earlier. Figure 6 shows the results. For small $\chi N$, the interface width becomes large relative to the core region, and solutions cease to exist. The structure of this relationship suggests a saddle-node bifurcation relative to $\chi N$.

An exhaustive search for single-peak solutions was performed over a wide range of $\chi N$ and $f$, where $\gamma$ is calibrated as described above. Figure 7 indicates where solutions were found relative to the spinodal curve (solid curve). The interval of existence is large for small volume fraction, whereas no steady states were found above $f \approx .2$, similar to the findings of Wang, Wang, and Yang [42] for spherical micelles in self-consistent field theory calculations.

Somewhat surprisingly, however, no steady solutions were found near the instability threshold. Rather than the solution relaxing to a single peak, a dynamical process of self-replication unfolds in a narrow region in parameter space (Figure 7). This phenomenon is discussed in what follows.

3.1.4. A second bifurcation and nonexistence near the instability threshold. An important ingredient in the explanation of the self-replication dynamics is the sudden disappearance of single-peaked and multipeaked steady solutions through 


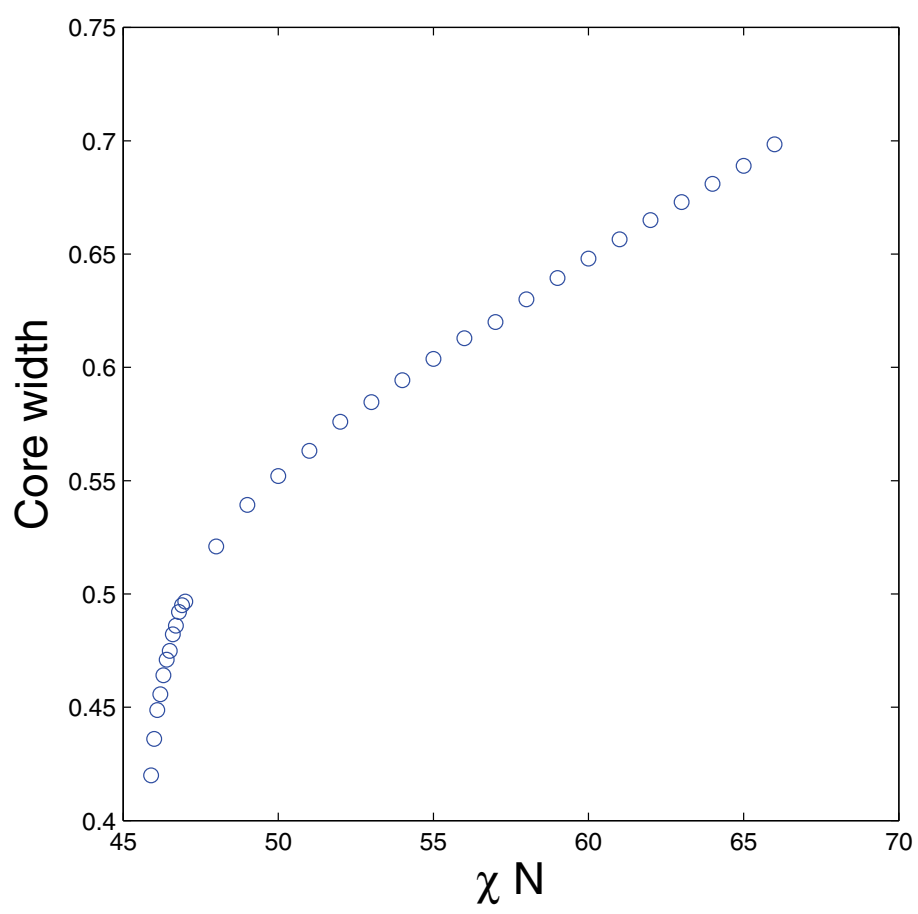

FIG. 6. The core width for solutions for $f=.1$ and $\gamma=71$ as a function of $\chi N$. Solutions exist only over a range below the spinodal instability and above a threshold determined by a crossover in core and interface sizes. The structure of this plot suggests a saddle-node bifurcation as a function of $\chi N$.

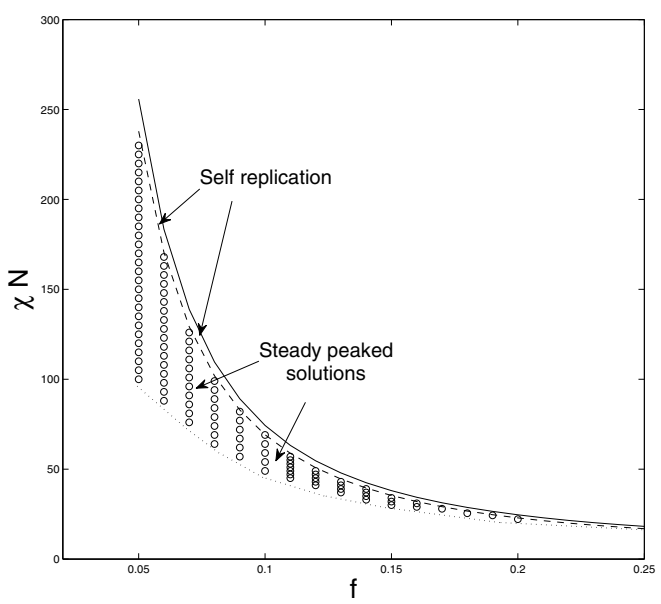

FIG. 7. Phase diagram for behavior in one dimension. The solid line is the spinodal curve, above which the homogeneous state is unstable. The region between the dotted and dashed lines is where steady, single-peak solutions were found, represented by circles. Between the solid and dashed lines, self-replicating behavior was observed. 
a bifurcation as $\chi N$ is increased. Dynamically, this is seen as a sudden growth of a peak at the location of the second largest local maximum as $\chi N$ increases past the bifurcation value $\chi N_{b}$. The origin of this growth appears to be related to the far field behavior given by (3.7). The location of the secondary (next largest) local maximum is roughly one wavelength $2 \pi /[\operatorname{Im} Q]$ from the global maximum; as a consequence, the value of this maximum can be approximated as $\phi_{\max } \approx f+C \exp (-2 \pi[\operatorname{Re} Q] /[\operatorname{Im} Q])$, where $C$ is some order one constant. When $\chi N$ is only slightly below the instability threshold $s(f)$, one can see from (3.5) that the real part of $Q$ is small, and the secondary maximum is therefore relatively large, that is, $\phi_{\max } \gg f$. There seems to be a threshold for the amplitude of this secondary maximum, above which growth will occur until another fully formed peak appears. As a consequence, when there is not enough spatial decay in the oscillating tail region, any large-amplitude peaked solution cannot be steady. Making this argument precise will be left to a future publication.

Numerical investigations provide some detail about solution's behavior just prior to the bifurcation. Steady solutions were tracked by continuation in the parameter $\chi N$. The relevant part of the spectrum of the linearization of (2.14) around the stable solution $\phi_{0}$ and corresponding eigenfunction were also computed using the Jacobian of the finite difference discretization. The near-translation invariance of $\phi_{0}$ gives one exponentially small eigenvalue (corresponding to the translation eigenfunction $\left.\sim d \phi_{0} / d x\right)$ and an otherwise stable point spectrum. Of greatest interest is the eigenvalue with greatest real part in the latter set. The behavior of these eigenvalues near the bifurcation value $\chi N_{b}$ is shown in Figure 8 for three different values of composition $f$. The square-root behavior of the eigenvalues is consistent with a saddle-node bifurcation of solutions.

The corresponding discrete eigenfunctions were also computed. These appear to come in pairs as a result of the left-right symmetry of the underlying steady solutions. Figure 9 shows a solution very close to the bifurcation, together with the critical eigenfunctions. The structure of the eigenfunctions is relevant in the later discussion of self-replication behavior that occurs beyond the bifurcation.

3.2. Cylindrical and spherical micelles. We now investigate isolated equilibria in two and three dimensions. Axisymmetric two-dimensional solutions correspond to cylindrical micelles, and radially symmetric three-dimensional solutions correspond to spherical micelles. The structure of the solutions is similar to the one-dimensional setting, with an $A$-monomer-rich core surrounded by a diffuse interface separating the core from a $B$-monomer-rich corona.

In polar and spherical coordinates, a radially symmetric steady state in dimension $d$ solves

$$
r^{1-d}\left(r^{d-1}\left[-r^{1-d}\left[r^{d-1} \phi_{r}\right]_{r}+g_{\phi}(\phi ; \chi N)\right]_{r}\right)_{r}+\gamma(f-\phi)=0
$$

on the half-line $r>0$, subject to Neumann boundary conditions $\phi_{r}=0$ at 0 and $\infty$ (assuming suitable decay), and also subject to the compositional constraint

$$
\int_{0}^{\infty}(\phi(r)-f) r^{d-1} d r=0 .
$$

3.2.1. Asymptotic structure. A construction similar to that in section 3.1.1 can be used to highlight the architecture of micelle solutions and derive approximations to the core radius. The scaling regions are identical to the one-dimensional problem, with the interface located at $r=\gamma^{-1 / 2} \rho^{*}$ (where $\rho^{*}$ is analogous to $z^{*}$ and 


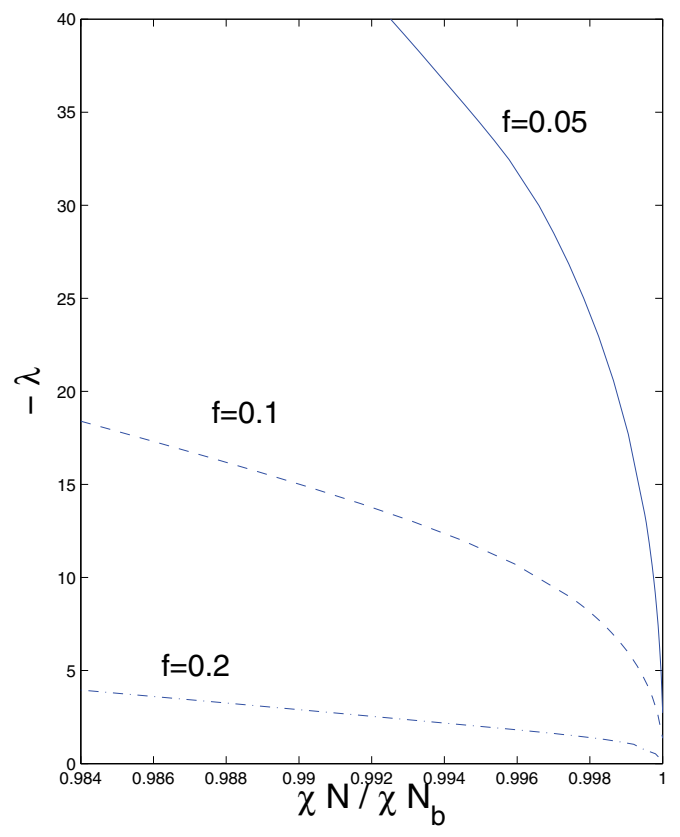

FIG. 8. Behavior of the principal eigenvalues (see text) $\lambda$ of the linearization of the dynamics computed numerically from the discretized operator. The bifurcation values are $\chi N_{b}=$ $244.15,71.194,25.314$ for $f=0.05,0.1,0.2$, respectively.

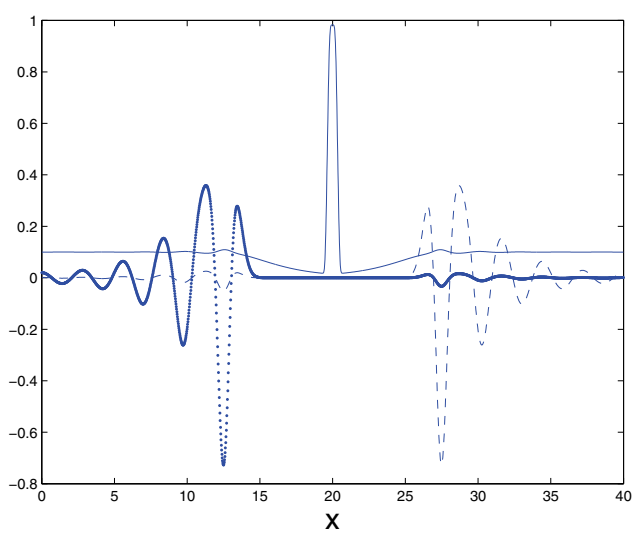

FIG. 9. A steady solution (solid) just at the bifurcation point $\chi N=71.19, f=0.1$, and a pair of discrete eigenfunctions (dotted and dashed) corresponding to the least unstable eigenvalue.

is determined by global considerations). The equation for $\phi$ in the interface region is to leading order in $\gamma^{-1 / 2}$ exactly the same as the one-dimensional problem. has

In the core and corona regions, using the rescaled coordinate $\rho=r / \gamma^{-1 / 2}$, one

$$
\left(\rho^{d-1} g_{\phi}(\phi ; \chi N)_{\rho}\right)_{\rho}+\rho^{d-1}(f-\phi)=0
$$


subject to the matching and boundary conditions

$$
\phi_{\rho}(0)=0, \quad \lim _{\rho \rightarrow \rho^{*}-} \phi=\phi_{+}, \quad \lim _{\rho \rightarrow \rho^{*}+} \phi=\phi_{-}, \quad \lim _{\rho \rightarrow \infty} \phi=f .
$$

As before, for quantitative purposes a simplification of problem (3.26) using the quadratic approximation of the potential (3.17) is enlightening, giving

$$
\beta^{2}\left(\rho^{d-1} \phi_{\rho}\right)_{\rho}+\rho^{d-1}(f-\phi)=0 .
$$

In two dimensions the solution is

$$
\phi= \begin{cases}f+\left(\phi_{-}-f\right) I_{0}(\rho / \beta) / I_{0}\left(\rho^{*} / \beta\right), & 0<\rho<\rho^{*}, \\ f+\left(\phi_{+}-f\right) K_{0}(\rho / \beta) / K_{0}\left(\rho^{*} / \beta\right), & \rho>\rho^{*},\end{cases}
$$

where $I_{0}, K_{0}$ are the usual modified Bessel functions. The solution in three dimensions is

$$
\phi= \begin{cases}f+\left(\phi_{-}-f\right) i_{0}(\rho / \beta) / i_{0}\left(\rho^{*} / \beta\right), & 0<\rho<\rho^{*}, \\ f+\left(\phi_{+}-f\right) k_{0}(\rho / \beta) / k_{0}\left(\rho^{*} / \beta\right), & \rho>\rho^{*},\end{cases}
$$

where the modified spherical Bessel functions are $i_{0}(r)=\sinh (r) / r$ and $k_{0}(r)=$ $\exp (-r) / r$. To determine $\rho^{*}$, we integrate (3.26) and use the constraint (3.25). This implies the relation

$$
\frac{f-\phi_{-}}{\phi_{+}-f}=B_{d}\left(\rho^{*} / \beta\right),
$$

where $B_{2}(x)=-I_{0}^{\prime}(x) K_{0}(x) /\left[K_{0}^{\prime}(x) I_{0}(x)\right]$ and $B_{3}(x)=-i_{0}^{\prime}(x) k_{0}(x) /\left[k_{0}^{\prime}(x) i_{0}(x)\right]$.

A further approximation for (3.31) can be made by linearizing (somewhat crudely but effectively) $B_{d}(x) \approx \alpha_{d} x$, where $\alpha_{2} \approx 0.3$ and $\alpha_{3} \approx 0.2$. For small $f$ this leads to a similar expression for the scaled core radius:

$$
\rho^{*}=\frac{\beta}{\alpha_{d}}\left(f-\phi_{-}\right) .
$$

This is consistent with the one-dimensional result where $\alpha_{1}=1$. The main conclusions to be drawn from the estimates (3.32) are that the core size scales with volume fraction $f$ and $\beta$ (which can be shown to be roughly proportional to $\chi N$ ) and that it increases with dimension.

3.2.2. Numerical solutions. To illustrate the nature of higher dimensional equilibria, the gradient flow dynamics (2.14) were used as before to numerically compute steady states. Figure 10 shows cylindrical and spherical solutions for $f=.1$ over a range of $\chi N$.

The prediction of core radius (3.32) is roughly in agreement with the numerical solutions. In particular, for a given value of parameters, the core size increases with dimension. Accepting the heuristic notion that micelle-type solutions exist when the core size is large compared to the interface width, this means that solutions will exist over a greater range of $\chi N$ as the dimension increases. This seems to be born out by our calculations: for $f=.1$, nontrivial solutions were found for $\chi N$ as small as 28 in two dimensions and 22 in three dimensions. 

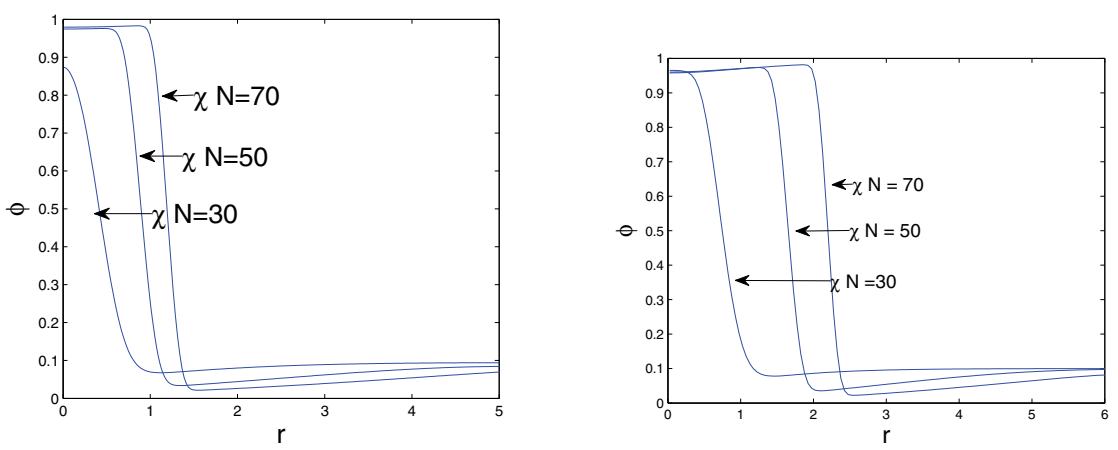

Fig. 10. Numerical solutions in two and three dimensions for $f=0.1$ and for various $\chi N$. Shown are composition profiles for two-dimensional cylindrical micelles (left) and three-dimensional spherical micelles (right).

4. Multipeaked solutions and self-replication. As in a variety of reaction diffusion problems $[2,10,44]$, the far field exponential decay of single-peak solutions allows for a study of the interaction of many such peaks. In particular, steady, multipeaked configurations are found. A related phenomenon is that of self-replication, where bifurcations of the underlying stable solutions can lead to space-filling pattern propagation. We provide evidence that this phenomenon is an example of the Nishiura-Ueyama self-replication mechanism [29].

4.1. Dynamics of multipeaked solutions. To construct a more complete description of the dynamics of (2.14), we now consider the behavior of an approximate superposition of isolated peaked equilibria. We suppose here that only stable solutions are being superposed, so that on long timescales, the isolated peaks remain intact. This idea appears to be robust in one dimension, whereas in higher dimensions instabilities can arise (see the discussion in section 5 ) that might invalidate this approach. We nevertheless present the analysis for arbitrary dimension.

For a superposition of single-peaked solutions, let $x_{i}$ denote the individual local maxima. Providing these are far enough apart, the dynamics $\phi_{t}$ should be transcendentally slow since only the tails of these structures deviate from equilibrium. This weak interaction results in the apparent translational motion. This is a common theme in the study of weakly interacting "pulse"-type solutions to PDEs, and the dynamics may be ascertained through perturbation methods [10,44].

The procedure outlined by Elphick, Meron, and Spiegel [10] can be used to reduce the dynamics to a set of coupled ODEs. As this work has been widely cited, we present only the details which are relevant for our problem. The perturbation parameter is $\epsilon=\exp (-(\operatorname{Re} Q) L)$, where $L$ is the characteristic distance between isolated peaks. The analysis assumes that $L$ is large, which is to say that peaks are well separated. The solution is written in terms of the slow timescale $\tau=\epsilon t$ as

$$
\phi(x, \tau)=f+\sum_{i=1}^{n}\left(\phi_{0}\left(x-x_{i}(\tau)\right)-f\right)+\epsilon \psi(x, \tau)
$$

where $\psi$ is a correction term and $\phi_{0}$ is a single equilibrium solution with its maximum 
at $x=0$, solving

$$
\begin{aligned}
& -a \Delta^{2} \phi_{0}+\Delta\left[g^{\prime \prime}(f)\left(\phi_{0}-f\right)+N\left(\phi_{0}\right)\right]+\gamma\left(f-\phi_{0}\right)=0, \\
& \quad N(\phi) \equiv g^{\prime}(\phi)-g^{\prime \prime}(f)(\phi-f)-g^{\prime}(f) .
\end{aligned}
$$

Note that $g^{\prime}()$ has been split into linear and nonlinear parts, reflecting the fact that only the nonlinear terms ultimately contribute to the interaction. Substitution of (4.1) into the dynamical equation (2.14) and making use of (4.2) leads to a linear equation for $\psi$ at order $\epsilon$,

$$
\begin{aligned}
\mathcal{L} \psi & =-\sum_{i=1}^{n} \nabla \phi_{i} \cdot\left(d x_{i} / d \tau\right)-\epsilon^{-1} \Delta R \\
R & \equiv N\left(f+\sum_{i=1}^{n}\left(\phi_{i}-f\right)\right)-\sum_{i=1}^{n} N\left(\phi_{i}-f\right),
\end{aligned}
$$

where the shorthand $\phi_{i}=\phi_{0}\left(x-x_{i}(\tau)\right)$ has been used and the linear operator is

$$
\mathcal{L} \equiv-a \Delta^{2}-\gamma+\Delta\left[g^{\prime \prime}\left(f+\sum_{i=1}^{n}\left(\phi_{i}-f\right)\right)\right.
$$

As explained in [10], the $R$ term is actually $\mathcal{O}(\epsilon)$.

The usual idea at this point is to invoke a Fredholm-type solvability argument that uses the exact zero eigenfunction for the adjoint of $\mathcal{L}^{\prime}$. It is consistent with the order of approximation, however, to just use an approximate version which involves the eigenfunctions $\eta$ satisfying

$$
-a \Delta^{2} \eta+g^{\prime \prime}\left(\phi_{0}\right) \Delta \eta-\gamma \eta=0
$$

together with an appropriate decay condition for $|x| \rightarrow \infty$. Construction of these eigenfunctions proceeds as follows. First, since $\phi_{0}$ is stationary with respect to the functional (2.1), it solves

$$
-a \Delta \phi_{0}(x)+g^{\prime}\left(\phi_{0}(x)\right)-\gamma \int G\left(x-x^{\prime}\right)\left[\phi\left(x^{\prime}\right)-f\right] d x^{\prime}=0 .
$$

Differentiation implies that any derivative $\omega_{k}=\partial \phi_{0} / \partial x_{k}$ solves the "linearization"

$$
-a \Delta \omega_{k}(x)+g^{\prime \prime}\left(\phi_{0}(x)\right) \omega_{k}(x)-\gamma \int G\left(x-x^{\prime}\right) \omega_{k}\left(x^{\prime}\right) d x^{\prime}=0 .
$$

Now let $\eta_{k}$ be the unique exponentially decaying solution to the Poisson problem

$$
\Delta \eta_{k}=\omega_{k}, \quad \lim _{|x| \rightarrow \infty} \eta_{k}=0 .
$$

Inserting (4.8) into (4.7) shows that $\eta_{k}$ solves (4.5). A couple of observations about the eigenfunctions prove useful. First, $\eta$ will have the same decay rate in the far field as $\phi_{0}$, that is, $\eta(x)=\mathcal{O}(\epsilon)$, when $|x| \gg L$. Second, $\eta_{k}$ inherits symmetry properties from $\partial \phi_{0} / \partial x_{k}$ : it is odd in the $x_{k}$ variable and even in the other variables.

The sufficiency of (4.5) can be demonstrated by taking the $L^{2}$ inner product of $\mathcal{L} \psi$ with some $\eta_{k}\left(x-x_{l}\right)$. After integrating by parts, one has

$$
\int \mathcal{L} \psi \eta_{k}\left(x-x_{l}\right) d x=\int \psi\left[-a \Delta^{2} \eta_{k}-\gamma \eta+g^{\prime \prime}\left(f+\sum_{i=1}^{n}\left(\phi_{i}-f\right)\right) \partial \phi_{l} / \partial x_{k}\right] d x
$$


where integrals are over $\mathbb{R}^{d}$. Since $\partial \phi_{l} / \partial x_{k}=\mathcal{O}(\epsilon)$ when $\left|x-x_{l}\right|>L$ and $\phi_{j}-f=\mathcal{O}(\epsilon)$ for $\left|x-x_{l}\right|<L$ when $j \neq l$, it follows that

$$
g^{\prime \prime}\left(f+\sum_{i=1}^{n}\left(\phi_{i}-f\right)\right) \partial \phi_{l} / \partial x_{k}=g^{\prime \prime}\left(\phi_{l}\right) \partial \phi_{l} / \partial x_{k}+\mathcal{O}(\epsilon) .
$$

By the definition of the eigenfunction (4.5), it follows that $\int \mathcal{L} \psi \eta_{k}\left(x-x_{l}\right) d x=\mathcal{O}(\epsilon)$.

Now taking inner products of (4.3) with $\eta_{k}\left(x-x_{l}\right), l=1, \ldots, n$, the order one terms are (in the original timescale)

$$
\frac{d x_{l}^{k}}{d t} \cdot \sum_{i=1}^{n}\left(\int \nabla \phi_{i} \eta_{k}\left(x-x_{l}\right) d x\right)=-\int R \frac{\partial \phi_{l}}{\partial x_{k}} d x
$$

where integration by parts and (4.8) were used.

By virtue of symmetries and spatial decay of $\eta_{k}$, only the term involving $\partial \phi_{l} / \partial x_{k}$ on the left-hand side survives at leading order. The right-hand side can be simplified considerably by noting that $\partial \phi_{l} / \partial x_{k}$ is order one only where $\left|x-x_{l}\right|<L$. Within this region, the approximations

$$
\begin{aligned}
N\left(f+\sum_{i=1}^{n}\left(\phi_{i}-f\right)\right) & =N\left(\phi_{l}\right)+\sum_{i \neq l} N^{\prime}\left(\phi_{l}\right)\left(\phi_{i}-f\right)+\mathcal{O}\left(\epsilon^{2}\right), \\
\sum_{i=1}^{n} N\left(\phi_{i}\right) & =N\left(\phi_{l}\right)+\mathcal{O}\left(\epsilon^{2}\right)
\end{aligned}
$$

hold by virtue of $N(f)=N^{\prime}(f)=0$ and $\left|\phi_{i}-f\right|=\mathcal{O}(\epsilon)$ for $i \neq l$. Therefore,

$$
R \frac{\partial \phi_{l}}{\partial x_{k}}=\frac{\partial \phi_{l}}{\partial x_{k}} \sum_{i \neq l} N^{\prime}\left(\phi_{l}\right)\left(\phi_{i}-f\right)+\mathcal{O}\left(\epsilon^{2}\right)
$$

and (4.11) reduces to

$$
\frac{d x_{l}^{k}}{d t}=-\sum_{i \neq l} \frac{\int N^{\prime}\left(\phi_{l}\right)\left(\phi_{i}-f\right)\left(\partial \phi_{l} / \partial x^{k}\right) d x}{\int\left(\partial \phi_{l} / \partial x^{k}\right) \eta_{k}\left(x-x_{l}\right) d x}
$$

Some general observations about the structure of (4.15) are illuminating. The system (4.15) describes the motions of the multipeaked solution as a closed, interacting particle system. The principal contribution to the integrals in the numerator involve one $\mathcal{O}(1)$ factor and another which is exponentially small. In the case where the tails of $\phi_{0}$ are strictly decaying, the sign of these integrals is unaffected by the relative distance of the peaks. This means that individual peaks would appear to attract or repel (repelling behavior has been noted in numerical experiments, but this case is not pursued here). On the other hand, when $\phi_{0}$ oscillates in the far field, the sign of the integrals also oscillates with the relative distances between peaks. This combination of attraction and repulsion should in general lead to stable arrangements; i.e., there exist steady multipeaked solutions. Such a situation is a precursor to the self-replication phenomenon, which is reported and discussed later. 

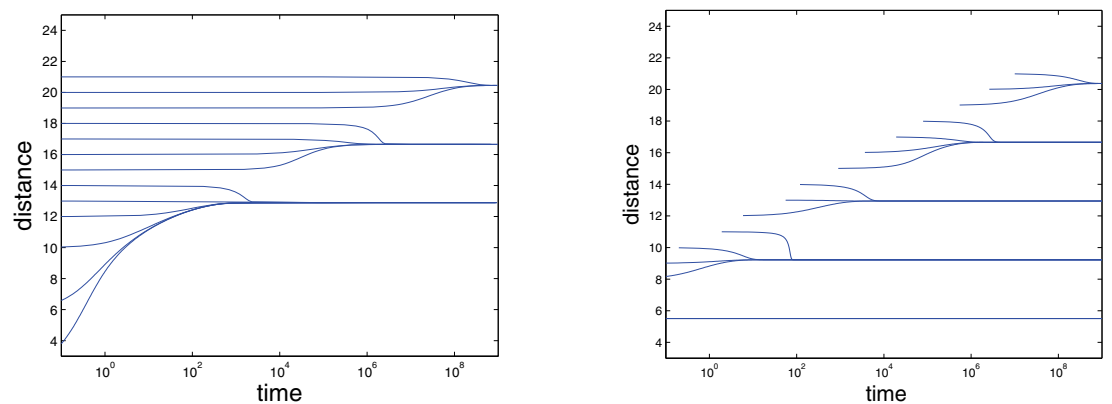

FIG. 11. Left: PDE simulation of two superposed peaks $(f=.1$ and $\chi N=60)$, showing the relative distance between peaks (local maxima) as a function of time. Right: Simulation of the reduced system (4.17). The approximation fails to capture the short-range repulsive dynamics but otherwise reproduces the pattern forming behavior.

4.1.1. Further simplification in one dimension. Specializing to one dimension, the far field behavior of equilibria (3.7) can be written as

$$
\phi_{0}-f \sim \operatorname{Re}[A \exp (-Q x)], \quad x \rightarrow \infty,
$$

where $A$ may be complex. Since the numerator of (4.15) has an order one factor and a factor which can be approximated by the exponentially small tail of $\phi_{0}$, for $x_{l}>x_{j}$ this integral approximates to

$$
\begin{aligned}
& \operatorname{Re}\left[\int A \exp \left(-Q\left(x-x_{j}\right)\right) N^{\prime}\left(\phi_{l}\right)\left(\partial \phi_{l} / \partial x^{k}\right) d x\right]=\operatorname{Re}\left[A I \exp \left(Q\left(x_{j}-x_{l}\right)\right)\right] \\
& I \equiv \int \exp (-Q x) N^{\prime}\left(\phi_{0}\right)\left(\partial \phi_{0} / \partial x^{k}\right) d x
\end{aligned}
$$

Furthermore, all these integrals are $\mathcal{O}\left(\epsilon^{2}\right)$ except for those involving the nearest neighbors $\left(x_{l+2}\right.$, for example, is a distance $\sim 2 L$, so that $\phi_{l+2}=\mathcal{O}\left(\epsilon^{2}\right)$ near $\left.x_{l}\right)$. The dominant terms in (4.15) may finally be written as (supposing $x_{l}$ are indexed from left to right)

$$
\frac{d x_{l}}{d t}=F\left(x_{l+1}-x_{l}\right)-F\left(x_{l}-x_{l-1}\right), \quad F(x)=\operatorname{Re}[J \exp (-Q x)],
$$

where the complex coefficient $J$ is

$$
J \equiv \frac{A I}{\int \phi_{0}^{\prime}(x) \eta(x) d x} .
$$

As an illustration, consider the interaction of two peaks. The full PDE dynamics were simulated for different initial spacings with $\chi N=60$ and $f=.1$. The results are shown in Figure 11. At small initial distance, the interaction appears to be strictly repulsive, whereas for large distances it is oscillatory. To make a comparison, the system (4.17) was also simulated. From the single-peak solution, we find $Q=$ $1.61+1.69 i$ and $A=48.3+75.9 i$. The profile for $\phi_{0}$ and the eigenfunction $\eta$ were computed numerically, which gives a coefficient value $J \approx(0.48+4.0 i) \times 10^{5}$. As would be expected, the perturbation calculation (also Figure 11) adequately captures the 


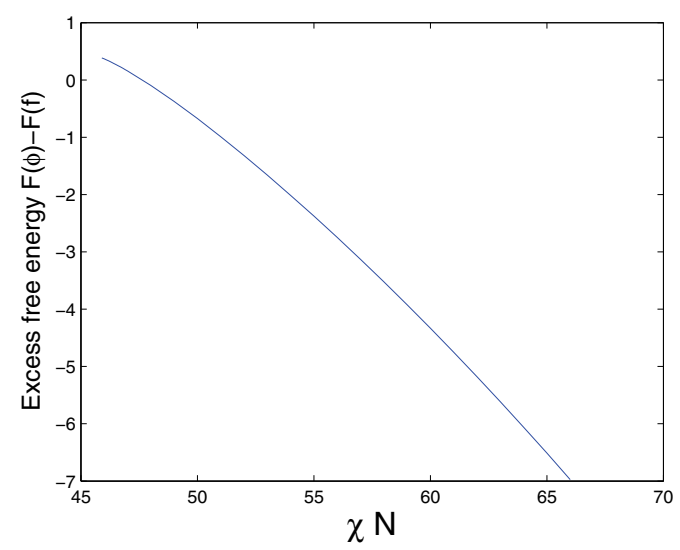

FIG. 12. Free energy of bilayer equilibria relative to the homogeneous state.

dynamics when equilibria are far apart but fails to predict the repulsive behavior when they are close. There is evidently a whole family of stable two-peak arrangements, characterized by displacements which are multiples (modulo a phase shift) of the underlying wavelength associated with the far field oscillation of a single-peak solution.

4.2. Free energy of multipeaked solutions. A useful quantity to evaluate is the energy of isolated peaked equilibria relative to the homogeneous state. In a situation where the energy is purely a local functional, the total energy is roughly the sum of the energies of individual equilibria up to transcendental corrections. This happens to be the case here for steady solutions, even though the energy is partly nonlocal. Indeed, letting $\psi_{x x}=f-\phi$ subject to Neumann boundary conditions, the nonlocal term can be written as

$$
\begin{aligned}
\frac{\gamma}{2} \int_{-\infty}^{\infty} \psi_{x}^{2} d x & =-\frac{\gamma}{2} \int_{-\infty}^{\infty} \psi(f-\phi) d x \\
& =\frac{1}{2} \int_{-\infty}^{\infty} \psi\left(-\phi_{x x}+g_{\phi}(\phi ; \chi N)\right)_{x x} d x \\
& =\frac{1}{2} \int_{-\infty}^{\infty}(f-\phi)\left(-\phi_{x x}+g_{\phi}(\phi ; \chi N)\right) d x .
\end{aligned}
$$

In other words, the nonlocal energy can be expressed as the sum of the energies on each subinterval that contains a peak. This argument can also be extended to higher dimensions.

It follows that if the excess free energy is negative, one might expect a propensity toward the development of more spatially localized equilibria. This is a necessary but not sufficient condition: If the excess energy is positive, the gradient flow cannot generate a state with even higher energy (this is of course not true if one also considers random fluctuations). In the case that it is negative, the question is more subtle, and the dynamical pathway to a lower energy state with many isolated equilibria could take the form of simple relaxation, nucleation via fluctuations, or front propagation. We investigate the latter below.

We have computed the excess free energy of one-dimensional bilayer solutions, that is, the value (2.1) relative to the homogeneous state $\phi=f$. Figure 12 shows the 


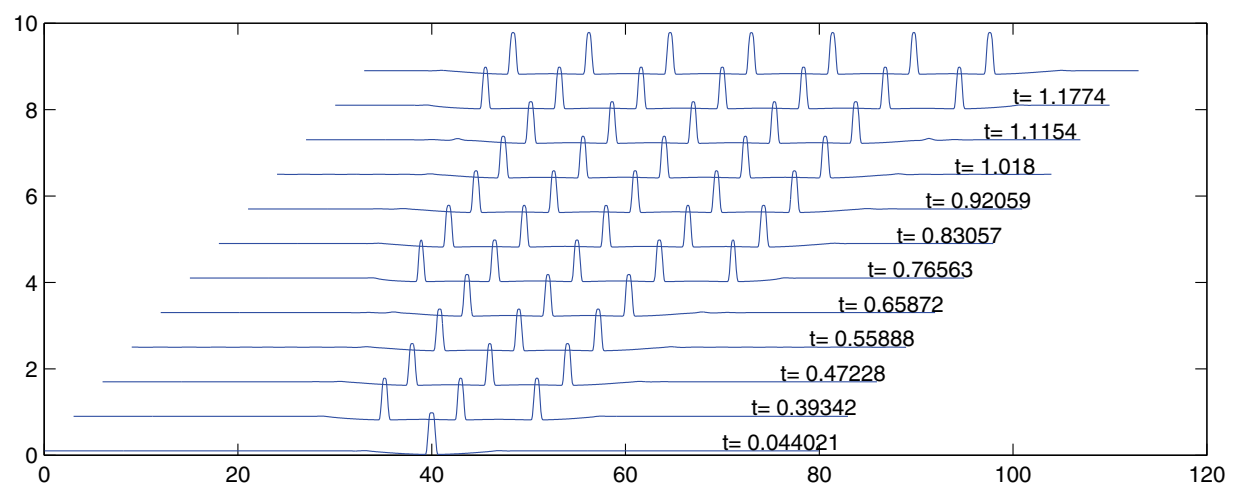

FIG. 13. Simulation for $f=.1$ and $\chi N=71.5$, just below the threshold for spinodal instability. New peaks are nucleated from oscillations in the tail region. Once fully formed, the process repeats, leading to continual self-replication.

free energy for various $\chi N$ with $f=0.1$ and $\gamma$ at its calibrated value. The excess is positive in a small range between the critical value $\chi N \approx 46$ but is negative over a much larger range. The energetic preference to bilayer equilibria also plays out in higher dimensions. It is connected to the (nonlinear) instability of two-dimensional micelle solutions. We investigate this in section 5.1.

4.3. Self-replication. The loss of exponentially decaying steady solutions as $\chi N$ is increased past the second bifurcation point $\chi N_{b}$, together with the requirement of ever-decreasing energy, means that a patterned state must propagate indefinitely. This propagation takes the form of replication of individual structures on either side of the multipeaked complex. As discussed in section 3.1.4, this occurs by nucleation in the tail region at the location of local maxima. A numerical example is shown in Figure 13.

There is a considerable body of literature of self-replicating patterns in reactiondiffusion systems (e.g., $[29,32,34])$. A general theory for this phenomenon was proposed by Nishiura and Ueyama [29], which appears to be applicable here. Their idea relies on the disappearance of all multipeaked solutions via saddle-node (or fold) bifurcation, more or less simultaneously. Self-replication dynamics are an "aftereffect" of this bifurcation, whereby the remnants of the unstable manifolds at the bifurcation point form a pathway leading from $N$-peak states to $(N+1)$-peak states. Many of these ingredients have been verified for particular systems, such as the Brusselator model [21].

For the present model, the results of section 4.1.1 suggest that existence of a onepeak solution is sufficient to guarantee multipeaked solutions. The converse question is more subtle. It was conjectured in section 3.1.4 that the spatial decay is the deciding factor in existence of steady solutions. Then all multipeaked solutions would, perhaps up to transcendentally small corrections, experience the bifurcation at the same value of $\chi N$, since they all have the same decaying oscillations given by (3.7).

The structure of the least stable eigenfunctions computed at the bifurcation point (see Figure 9) provides a clue to the nature of the unstable manifold that leads away from the bifurcation point. Their structure suggests a dynamical pathway that leads to growth of peaks on either side of an existing peak, consistent with numerical obser- 


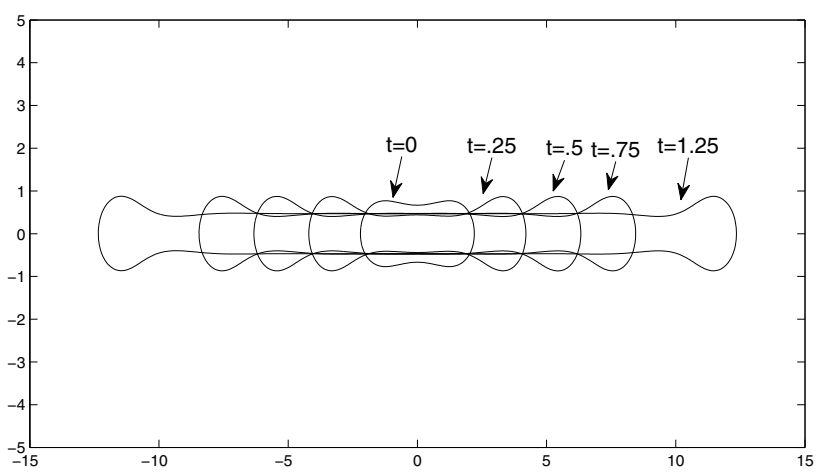

FIG. 14. Growth of a filament $(\chi N=60, f=.1)$ in two dimensions that begins as an elongated perturbed cylindrical micelle. The curves correspond to the contour $\phi=1 / 2$. The filament crosssection is essentially the same as the corresponding bilayer solution.

vation. Note this is different from the even-symmetry "dimple"-shaped eigenfunction that leads to pulse splitting in other self-replication problems $[8,21]$.

5. Illustrations of higher dimensional dynamics. Although the focus of this paper is largely about equilibria and one-dimensional dynamics, some preliminary results on dynamics in two dimensions show how the forgoing analysis connects to the larger questions of block copolymer self-assembly in the disordered regime. The results here are principally numerical in nature, as the simulations show that a complete characterization of the system's evolution is a challenging question.

5.1. Filamentary instability of cylindrical micelles and bilayer formation. In two dimensions, one might expect a competition between cylindrical (i.e., circular) and bilayer (i.e., linear-shaped) equilibria. Even when cylindrical solutions are stable, it is expected that large perturbations might favor a filamentary structure (we use the term "filament" since the numerically observed two-dimensional patterns are long, narrow domains; in three dimensions, these would correspond to bilayer sheets of finite extent) whose cross-sections are roughly one-dimensional equilibria. Such a scenario has been observed in simulations (Figure 14), where the ends of the filament propagate outward in a steady fashion.

The propagation of the filamentary ends is related to the excess free energy of a bilayer solution. If the excess energy is negative, then there should be a relative energy benefit for the filament to grow, assuming the cross-section is essentially a onedimensional equilibrium. Of course, this might be a little too simplistic: the energy (2.1) is nonlocal, and thus the energy per unit length is somewhat affected by the presence of filament ends in addition to other structures that may be nearby.

In the case where several initially cylindrical micelles compete, the result is dramatic (Figure 15). The interaction of nearby filaments disrupts their growth, causing either inhibition or occasionally "tip splitting." Filaments may bend in response to other nearby structures and occasionally break by pinching. This process goes on for some time until the entire domain is filled.

5.2. Self-replication in higher dimensions. The self-replication phenomenon in higher dimensions has also been studied in the literature (e.g., [22]). We conclude 

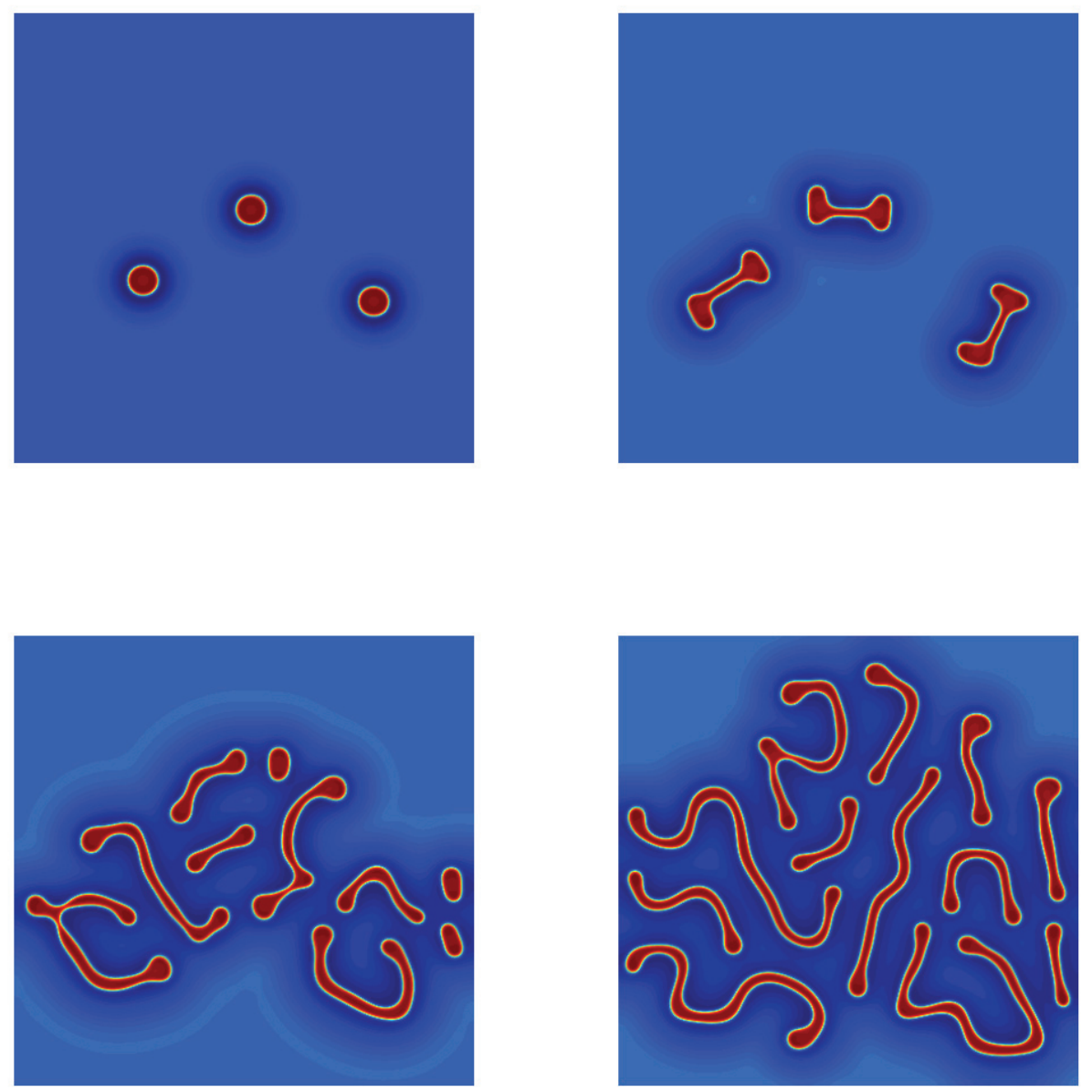

FIG. 15. Evolution of several competing filaments $(\chi N=60, f=.1)$ in two dimensions at times $t=0, .5,1.0,1.5$ (the domain size is $30^{2}$, and the red color indicates $\phi \approx 1$ ). The growing filament ends can split, and filaments bend to accommodate other nearby structures.

our discussion of dynamical phenomenon by demonstrating how this unfolds for the present model, using arguments analogous to those discussed in section 4.2. A complete analysis of this phenomena will be deferred to a future publication.

As for (one-dimensional) solutions, we have computed the excess free energy $F(\phi)-F(f)$ of equilibria both in two and three dimensions (Figure 16) for $f=.1$ and for $\chi N$ over the range of existence. As before, the excess energy is positive near the lower existence threshold but is negative over a wide range of $\chi N$.

The fact that the system can lower its energy by replication of micellar elements suggests that this behavior would be common. As in one dimension, however, we have found this dynamical phenomenon only for $\chi N$ near (but below) the spinodal 


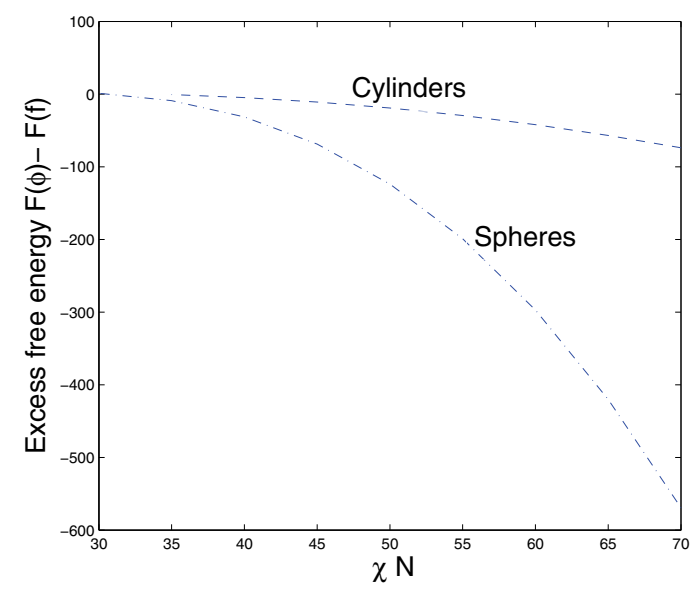

FIG. 16. Free energy of a two-dimensional cylindrical solution and three-dimensional spherical equilibria relative to the homogeneous state. In both cases $f=.1$.

transition. Figure 17 shows the evolution starting from a single "seed." The initial condition was $\phi=.9$ inside a circle of radius 1 and $\phi=.1$ outside. For somewhat smaller $\chi N$, such an initial condition would relax toward a single circular (cylindrical micelle) equilibrium. For $\chi N=70$, just below the point of spinodal instability, the pattern propagates in a radial fashion, producing concentric layers of circular micelles. Some of these are observed to lengthen via the filamentary growth mechanism. The ultimate fate of this assembly is complicated and involves interaction of the individual elements via growth and coarsening.

6. Conclusion. We have provided evidence of nonperiodic equilibria in density functional models of diblock copolymers for a regime previously unexplored. In general, existence requires sufficiently large interaction $\chi N$ and small volume fraction $f$, although relaxation to steady solutions gives way to self-replication dynamics near the spinodal instability.

There are a variety of unanswered questions which will be addressed in forthcoming work, including the stability of equilibria, and verification of the arguments that describe the secondary bifurcation and self-replication. Dynamics in three dimensions have also not been fully explored. One expects to find a linear or nonlinear instability of spheres which produce cylinders with growing ends, analogous to the two-dimensional filamentary instability, or even growth in two directions that evolves into a bilayer sheet.

\section{Appendix.}

Verification of estimate (3.16). We observe that by a change of variables,

$$
\int_{z^{*}}^{\infty}(f-\phi(z)) d z=\int_{0}^{g_{\phi}(f)} \frac{f-\phi(\psi)}{d \psi / d z} d \psi
$$

Integrating (3.14) and using the convexity of $g^{*}$ gives

$$
\text { (A.2) } \frac{1}{2} \psi_{z}^{2}=g^{*}(\psi)-g^{*}\left(\psi_{f}\right)-f\left(\psi-\phi_{f}\right) \geq \frac{1}{2} \min _{\left[0, \psi_{f}\right]}\left(g^{*}\right)^{\prime \prime}(\psi)\left(\psi-\psi_{f}\right)^{2} \equiv C_{1}\left(\psi-\psi_{f}\right)^{2} .
$$



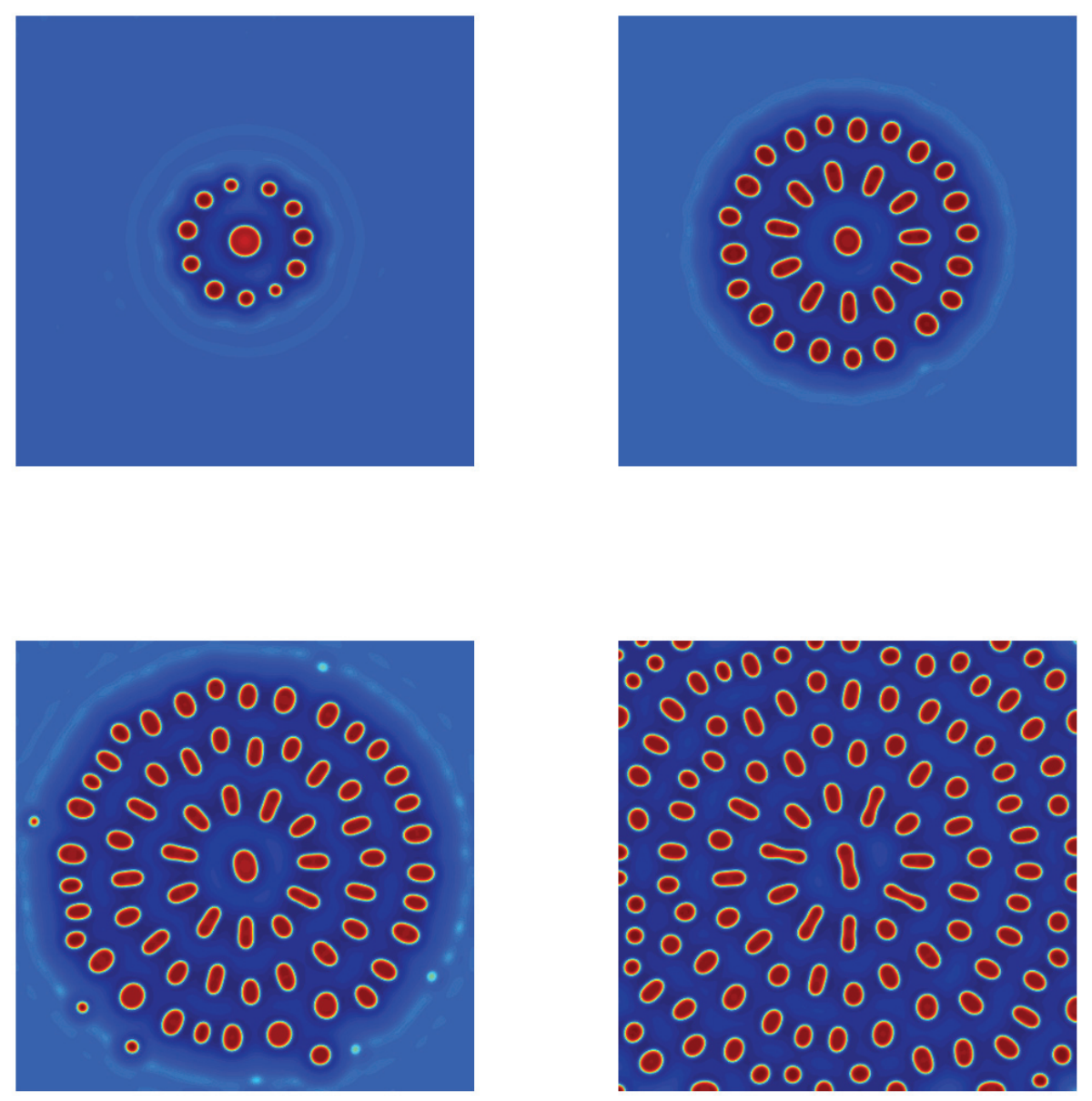

FIG. 17. Self-replication in two dimensions $(\chi N=70, f=.1)$. The evolution is shown at times $t=.14, .28, .42, .56$ (the other details are the same as in Figure 15). The initial data was a single circular micelle-type solution. Notice that micelle structures formed in the center elongate via the filamentary growth mechanism.

In addition, one can estimate

$$
\begin{aligned}
f-\phi(\psi) & \leq\left[\max _{\left[\phi_{-}, f\right]} \frac{d \phi}{d \psi}\right]\left(\psi_{f}-\psi\right) \equiv C_{2}\left(\psi_{f}-\psi\right), \\
\psi_{f} & \leq\left[\max _{\left[0, \psi_{f}\right]} \frac{d \psi}{d \phi}\right]\left(f-\phi_{-}\right) \equiv C_{3}\left(f-\phi_{-}\right) .
\end{aligned}
$$

Combining ( A.1), (A.2), and (A.3) gives

$$
\int_{z^{*}}^{\infty}(f-\phi(z)) d z \leq\left(\frac{C_{2} C_{3}}{C_{1}}\right)\left(f-\phi_{-}\right) .
$$


Proof of Proposition 3.1. Let $\phi$ be any admissible state (not just a local minimizer of $F$ ). Consider as test functions the rescalings

$$
\phi_{c}=c(\phi-f)+f
$$

By virtue of the volume constraint, the variational problem remains the same if we add any linear function of $\phi$ to the integrand of (2.1). Let $H(\phi)=h(\phi)-h^{\prime}(f) \phi$ so that by convexity of $H$,

$$
(\phi-f) H^{\prime}(\phi) \geq \frac{1}{2} \inf _{\phi \in[0,1]} h^{\prime \prime}(\phi)(\phi-f)^{2} .
$$

It is therefore equivalent to consider the modified functional

$$
\begin{aligned}
F\left(\phi_{c}\right)=\int \frac{c^{2} a}{2}|\nabla \phi|^{2} & +H\left(\phi_{c}\right)-c^{2} \chi N(\phi-f)^{2} d x \\
& +c^{2} \frac{\gamma}{2} \iint G\left(x-x^{\prime}\right)[\phi(x)-f]\left[\phi\left(x^{\prime}\right)-f\right] d x d x^{\prime} .
\end{aligned}
$$

Thus

$\frac{d F\left(\phi_{c}\right)}{d c} \geq 2 c \int \frac{a}{2}|\nabla \phi|^{2}-\beta(\phi-f)^{2} d x+\frac{\gamma}{2} \iint G\left(x-x^{\prime}\right)[\phi(x)-f]\left[\phi\left(x^{\prime}\right)-f\right] d x d x^{\prime}$,

where $\beta=\chi N-\frac{1}{2} \inf _{\phi \in[0,1]} h^{\prime \prime}(\phi)$. Letting $\hat{\phi}(k)$ be the Fourier transform of $\phi-f$, one can write this as

$$
\left.\frac{d F\left(\phi_{c}\right)}{d c}\right|_{c=0} \geq \int\left(\frac{a}{2} k^{2}-\beta+\gamma_{2} k^{-2}\right) \hat{\phi}(k)^{2} d k,
$$

which is positive if

$$
\chi N \leq \sqrt{\gamma a}+\frac{1}{2} \inf _{\phi \in[0,1]} h^{\prime \prime}(\phi) .
$$

In this case, $d F / d c>0$ unless $\phi \equiv f$, which is to say that $\phi \equiv f$ is the only minimizer.

Proof of Proposition 3.2. We construct a test function on $[0, \infty)$ which resembles the computed bilayer-type equilibria, and we assume reflection across $x=0$. For a given $\chi N$, let $\phi_{+}, \phi_{-}$be minimizers of $g(\phi ; \chi N)$ with $\phi_{-}<\phi_{+}$(we suppose $g$ necessarily has two wells for large enough $\chi N)$. Let

$$
\varphi= \begin{cases}\phi_{+}, & x<d_{1}, \\ \phi_{-}+\left(x-d_{1}\right)\left(\phi_{+}-\phi_{-}\right) / \xi, & d_{1}<x<d_{2}, \\ f-\left(f-\phi_{-}\right) \exp \left(-\left(x-d_{2}\right) / d_{3}\right), & x>d_{2},\end{cases}
$$

where $d_{1}, d_{2,3} \geq 0$ are to be prescribed to be consistent with the volume constraint, and $\xi=d_{2}-d_{1}$ plays the role of an interface width. Note that $d_{1}$ may be negative.

The energy of $\varphi$ relative to $\phi \equiv f$ can be written as

$$
F_{r e l}(\phi)=\int_{0}^{\infty} \frac{a}{2} \varphi_{x}^{2}+[g(\varphi ; \chi N)-g(f ; \chi N)]+\frac{\gamma}{2} \psi_{x}^{2} d x, \quad \psi_{x x}=f-\varphi,
$$

where the boundary conditions are $\psi_{x}=0$ at 0 and $\infty$. 
We first estimate the nonlocal term. For $0 \leq x \leq d_{1},\left|\psi_{x x}\right| \leq 1$ so that $\left|\psi_{x}\right| \leq x$. Thus the contribution to the energy on that interval has the bound

$$
\frac{\gamma}{2} \int_{0}^{d_{1}} \psi_{x}^{2} d x \leq \frac{\gamma d_{2}^{3}}{6}
$$

For $x>d_{2}$, one computes directly

$$
\frac{\gamma}{2} \int_{d_{2}}^{\infty} \psi_{x}^{2} d x=\frac{\gamma}{4} d_{3}^{3}\left(f-\phi_{-}\right)^{2}
$$

For $d_{1} \leq x \leq d_{2}$, one has $\left|\psi_{x}\right|<\left(f-\phi_{-}\right) d_{3}$, so that

$$
\frac{\gamma}{2} \int_{d_{1}}^{d_{2}} \psi_{x}^{2} d x \leq \frac{\gamma}{2} \xi d_{3}^{2}\left(f-\phi_{-}\right)^{2} .
$$

By virtue of the volume constraint (3.3),

$$
d_{1} \leq \frac{1}{\phi_{+}-f} \int_{0}^{d_{2}}(\varphi-f) d x=-\frac{1}{\phi_{+}-f} \int_{d_{2}}^{\infty}(\varphi-f) d x=d_{3} \frac{f-\phi_{-}}{\phi_{+}-f} .
$$

Combining this with (A.6), and adding estimates (A.7), (A.8), the entire nonlocal contribution has a bound

$$
\frac{\gamma}{2} \int_{0}^{\infty} \psi_{x}^{2} d x \leq C \frac{\gamma}{4} d_{3}^{3}\left(f-\phi_{-}\right)^{2}
$$

where the constant $C=1+\frac{2}{3}\left(f-\phi_{-}\right) /\left(\phi_{+}-f\right)^{3}+\xi / d_{3}$. Our choice of $d_{3}$ (see below) has $d_{3} \rightarrow \infty$ when $f \rightarrow 0$.

The next contribution to $F(\varphi)$ is the surface energy

$$
\int_{0}^{d_{2}} \frac{a}{2} \varphi_{x}^{2}+[g(\varphi)-g(f)] d x \leq \int_{0}^{d_{2}} \frac{a}{2} \varphi_{x}^{2}+\sigma_{1} \varphi(1-\varphi) d x \leq \frac{a}{2 \xi}+\frac{\sigma_{1} \xi}{6}=\frac{\sqrt{3}}{3} \sqrt{a \sigma_{1}}
$$

where $\xi=\sqrt{3 a / \sigma_{1}}$ is chosen to be optimal.

The local terms' contribution for $x>d_{2}$ is (A.11)

$\int_{d_{2}}^{\infty} \frac{a}{2} \varphi_{x}^{2}+[g(\varphi)-g(f)] d x \leq \int_{d_{2}}^{\infty} \frac{a}{2} \varphi_{x}^{2}+\sigma_{2}(\varphi-f) d x=\frac{a\left(f-\phi_{-}\right)^{2}}{4 d_{3}}-d_{3} \sigma_{2}\left(f-\phi_{-}\right)$.

In the limit $f \rightarrow 0$, the optimal choice of $d_{3}$ is determined by ignoring the $\phi_{x}^{2}$ term in (A.11) and is $d_{3}=\sqrt{\frac{4 \sigma_{2}}{3 \gamma\left(f-\phi_{-}\right)}}$. With this choice, combining (A.9), (A.10), and (A.11) and setting them $\leq 0$ gives the desired estimate (3.22).

Acknowledgments. The author has benefitted from conversations with Rustum Choksi and Glenn Fredrickson.

\section{REFERENCES}

[1] F. S. Bates And G. H. Fredrickson, Block copolymers-designer soft materials, Physics Today, 52 (1999), pp. 32-38.

[2] G. Bellettini And G. Fusco, Stable dynamics of spikes in solutions to a system of reactiondiffusion equations, Asymptot. Anal., 26 (2001), pp. 307-357. 
[3] Y. Bohbot-Raviv And Z.-G. Wang, Discovering new ordered phases of block copolymers, Phys. Rev. Lett., 85 (2000), pp. 3428-3431.

[4] S. A. Brazovskil, Phase transition of an isotropic system to a nonuniform state, J. Exp. Theor. Phys., 41 (1975), pp. 85-89.

[5] J. W. CAHn AND J. E. Hilliard, Free energy of a nonuniform system I: Interfacial free energy, J. Chem. Phys., 28 (1957), pp. 258-267.

[6] R. Choksi, M. A. Peletier, And J. F. Williams, On the phase diagram for microphase separation of diblock copolymers: An approach via a nonlocal Cahn-Hilliard functional, SIAM J. Appl. Math., 69 (2009), pp. 1712-1738.

[7] R. Choksi AND X. Ren, On the derivation of a density functional theory for microphase separation of diblock copolymers, J. Statist. Phys., 113 (2003), pp. 151-176.

[8] A. Doelman, W. Eckhaus, And T. J. Kaper, Slowly modulated two-pulse solutions in the Gray-Scott model I: Asymptotic construction and stability, SIAM J. Appl. Math., 61 (2000), pp. 1080-1102.

[9] E. E. Dormidontova And T. P. Lodge, The order-disorder transition and the disordered micelle regime in sphere-forming block copolymer melts, Macromolecules, 34 (2001), pp. 91439155.

[10] C. Elphick, E. Meron, and E. A. Spiegel, Patterns of propagating pulses, SIAM J. Appl. Math., 50 (1990), pp. 490-503.

[11] G. H. Fredrickson, The Equilibrium Theory of Inhomogeneous Polymers, Oxford Science Publications, Clarendon Press, Oxford, UK, 2006.

[12] G. H. Fredrickson and F. S. Bates, Dynamics of block copolymers: Theory and experiment, Ann. Rev. Materials Sci., 26 (1996), pp. 501-550.

[13] R. E. Goldstein, D. J. Muraki, and D. M. Petrich, Interface proliferation and the growth of labyrinths in a reaction-diffusion system, Phys. Rev. E (3), 53 (1996), pp. 3933-3957.

[14] I. W. Hamley, The Physics of Block Copolymers, Oxford Science Publications, Oxford, UK, 1998.

[15] T. Hashimoto, N. Sakamoto, And T. Koga, Nucleation and growth of anisotropic grain in block copolymers near order-disorder transition, Phys. Rev. E (3), 54 (1996), pp. 58325835.

[16] X. He AND F. Schmid, Spontaneous formation of complex micelles from a homogeneous solution, Phys. Rev. Lett., 100 (2008), 137802.

[17] E. HeLfand, Theory of inhomogeneous polymers: Fundamentals of the Gaussian random-walk model, J. Chem. Phys., 62 (1975), pp. 999-1005.

[18] E. Helfand and Y. Tagami, Theory of the interface between immiscible polymers, J. Polymer Sci. B, 9 (1971), pp. 741-746.

[19] K. M. Hong AND J. Nooland, Theory of inhomogeneous multicomponent polymer systems, Macromolecules, 14 (1981), pp. 727-736.

[20] D. IRON AND M. J. WARD, A metastable spike solution for a nonlocal reaction-diffusion model, SIAM J. Appl. Math., 60 (2000), pp. 778-802.

[21] T. Kolokolnikov, M. J. WARD, AND J. Wei, Self-replication of mesa patterns in reactiondiffusion systems, Phys. D, 236 (2007), pp. 104-122.

[22] T. Kolokolnikov, M. J. WARD, AND J. WeI, Spot self-replication and dynamics for the Schnakenburg model in a two-dimensional domain, J. Nonlinear Sci., 19 (2009), pp. 1-56.

[23] L. LEIBLER, Theory of microphase separation in block copolymers, Macromolecules, 13 (1980), pp. $1602-1617$.

[24] M. W. Matsen And F. S. Bates, Unifying weak- and strong-segregation block copolymer theories, Macromolecules, 29 (1996), pp. 1091-1098.

[25] M. W. Matsen And M. Schick, Stable and unstable phases of a diblock copolymer melt, Phys. Rev. Lett., 72 (1994), pp. 2660-2663.

[26] C. B. Muratov, Theory of domain patterns in systems with long-range interactions of Coulomb type, Phys. Rev. E (3), 66 (2002), 066108.

[27] C. B. Muratov and V. V. Osipov, Scenarios of domain pattern formation in a reactiondiffusion system, Phys. Rev. E (3), 54 (1996), pp. 4860-4879.

[28] Y. Nishiura and I. Ohnishi, Some mathematical aspects of the micro-phase separation of diblock copolymers, Phys. D, 84 (1995), pp. 31-39.

[29] Y. Nishiura And D. Ueyama, A skeleton structure of self-replicating dynamics, Phys. D, 130 (1999), pp. 73-104.

[30] T. OHTA AND K. KAWASAKI, Equilibrium morphology of block copolymer melts, Macromolecules, 19 (1986), pp. 2621-2632.

[31] T. Ohta AND K. KaWASAKI, Comment on the free energy functional of block copolymer melts in the strong segregation limit, Macromolecules, 23 (1990), pp. 2413-2414. 
[32] J. E. Pearson, Complex patterns in a simple system, Science, 261 (1993), pp. 189-192.

[33] R. L. Pego, Front migration in the nonlinear Cahn-Hilliard equation, Proc. Roy. Soc. London Ser. A, 422 (1989), pp. 261-278.

[34] W. N. Reynolds, J. E. Pearson, and S. Ponce-Dawson, Dynamics of self-replicating patterns in reaction diffusion systems, Phys. Rev. Lett., 72 (1994), pp. 2797-2800.

[35] N. Sakamoto, T. Hashimoto, C. D. Han, D. Kim, and N. Y. Vaidya, Order-order and order-disorder transitions in a polystyrene-block-polyisoprene-block-polystyrene copolymer, Macromolecules, 30 (1997), pp. 1621-1632.

[36] M. Schwab And B. Stühn, Thermotropic transition from a state of liquid order to a macrolattice in asymmetric diblock copolymers, Phys. Rev. Lett., 76 (1996), pp. 924-927.

[37] A. N. Semenov, Microphase separation in diblock copolymer melts: Ordering of micelles, Macromolecules, 22 (1989), pp. 2849-2851.

[38] A. N. Semenov, Theory of block copolymer interfaces in the strong-segregation limit, Macromolecules, 26 (1993), pp. 6617-6621.

[39] E. L. Thomas, D. M. Anderson, C. S. Henkee, and D. Hoffman, Periodic area-minimizing surfaces in block copolymers, Nature, 334 (1988), pp. 598-601.

[40] T. Uneyama AND M. DoI, Calculation of the micellar structure of polymer surfactant on the basis of the density functional theory, Macromolecules, 38 (2005), pp. 5817-5825.

[41] Y. van Gennip and M. A. Peletier, Copolymer-homopolymer blends: Global energy minimisation and global energy bounds, Calc. Var. Partial Differential Equations, 33 (2008), pp. $75-111$.

[42] J. WANG, Z.-G. WANG, AND Y. YANG, Nature of disordered micelles in sphere-forming block copolymer melts, Macromolecules, 38 (2005), pp. 1979-1988.

[43] X. Wang, E. E. Dormidontova, and T. P. LOdGe, The order-disorder transition and the disordered micelle regime for poly(ethylenepropylene-b-dimethylsiloxane) spheres, Macromolecules, 35 (2002), pp. 9687-9697.

[44] M. J. WARD, Metastable dynamics and exponential asymptotics in multi-dimensional domains, in Multiple-Time-Scale Dynamical Systems (Minneapolis, 1997), Springer, New York, 2001, pp. 233-259.

[45] M. J. WARD, Asymptotic methods for reaction-diffusion systems: Past and present, Bull. Math. Biol., 68 (2006), pp. 1151-1167. 